Ebralidze A.K. et al. text and figure legends

\title{
Formation and recycling of an active epigenetic mark mediated by cell cycle-
}

\section{specific RNAs}

Alexander K. Ebralidze ${ }^{1}$, Simone Ummarino ${ }^{1,3, \dagger}$, Mahmoud A. Bassal ${ }^{1,2,3, \dagger}$, Haoran Zhang ${ }^{1}$, Bogdan

Budnik $^{4}$, Emanuele Monteleone ${ }^{5}$, Dennis Kappei ${ }^{2}$, Yanjing V. Liu ${ }^{2}$, Danielle E.Tenen ${ }^{3}$, Rory

Coffey $^{1,3}$, Mee Rie Sheen ${ }^{1}$, Yanzhou Zhang ${ }^{1}$, Anaïs Wanet ${ }^{1,3}$, Bon Q. Trinh ${ }^{1,3}$, Valeria Poli ${ }^{5}$,

Vladimir Espinosa Angarica², Roberto Tirado Magallanes², Touati Benoukraf ${ }^{2,6}$, Colyn Crane-

Robinson $^{7}$, Annalisa Di Ruscio*1,8§, and Daniel G. Tenen*1,2,3 ${ }^{*}$.

$\uparrow$ These two authors equally contributed to the work.

*These two authors equally contributed to the work.

${ }^{1}$ Harvard Medical School Initiative for RNA Medicine, Harvard Medical School, Boston, MA, 02115, USA

${ }^{2}$ Cancer Science Institute, National University of Singapore, 117599, Singapore

${ }^{3}$ Harvard Stem Cell Institute, Harvard Medical School, Boston, MA, 02115, USA

${ }^{4}$ MSRRL, FAS, Division of Science, Harvard University, Cambridge, MA 02139, USA

${ }_{5}^{5}$ Department of Molecular Biotechnology and Health Sciences, and GenoBiToUS, Genomics and

Bioinformatics Service, University of Turin, Turin, 10126, Italy

${ }^{6}$ Discipline of Genetics, Faculty of Medicine, Memorial University of Newfoundland, St. John's, NL, A1B 3V6, Canada

${ }^{7}$ Biophysics Laboratories, University of Portsmouth, St. Michael's Building, Portsmouth, PO1 2DT, UK

${ }^{8}$ University of Eastern Piedmont, Department of Translational Medicine, Novara, 28100, Italy

${ }^{9}$ Cancer Research Institute, Beth Israel Deaconess Medical Center, Boston, 330 Brookline Avenue Boston,

MA 02215

${ }^{\S}$ Contact:

Daniel G. Tenen

Center for Life Sciences

3 Blackfan Circle

Room 437

Boston, MA 02115

Tel: 617-735-2235

FAX: 617-735-2222

daniel.tenen@nus.edu.sg

Annalisa Di Ruscio

Center for Life Sciences

3 Blackfan Circle

Room 437

Boston, MA 02115

Tel: 617-735-2217

FAX: 617-735-2222

adirusci@bidmc.harvard.edu 
Ebralidze A.K. et al. text and figure legends

\section{Abstract}

The mechanisms by which epigenetic modifications are established in gene regulatory regions of active genes remain poorly understood. The data presented show that the establishment and recycling of a major epigenetic mark, the acetylated form of the replacement histone H2A.Z, is regulated by cell cycle-specific long noncoding RNAs encoded in regions adjacent to the promoters of active genes. These transcripts, termed SPEARs (S Phase EArly RNAs), are induced in early $S$ phase: their expression precedes that of the downstream genes on which they exert their regulatory action. SPEARs drive the modification and deposition of the acetylated form of histone $\mathrm{H} 2 \mathrm{~A} . \mathrm{Z}$ by bringing together the replacement histone and the histone acetyl transferase TIP60. This widespread bimodal pathway constitutes a novel RNAmediated mechanism for the establishment of epigenetic marks and cell-specific epigenetic profiles, thereby providing a unifying explanation for the accuracy and persistence of epigenetic marks on chromatin.

A wealth of epigenomics data have recently provided meaningful biological and clinical information but little attention has been paid to the mechanisms for the establishment ('writing') and the maintenance of specific epigenetic patterns. Epigenetic aberrations are a frequent feature of deleterious conditions such as cancers and other genetic diseases ${ }^{1}$. In particular, subsets of histone variants and their post-translational modifications have emerged as prominent players among other epigenetic marks. The histone H2A.Z (the major H2A variant), for instance, is associated with gene activation $^{2-5}$ by means of the balance between the acetylated $v s$. the non-acetylated forms at the promoters of genes ${ }^{6-8}$. Acetylation of H2A.Z is catalyzed by the histone acetyltransferase TIP-60 subsequent to its inclusion within nucleosomes ${ }^{9}$ but it remains unexplained how the balance between the acetylated and unmodified forms is inherited and preserved. 
Ebralidze A.K. et al. text and figure legends

The concepts underlying epigenetic inheritance have recently undergone significant change. A number of studies demonstrated that epigenetic marks can cross generation borderlines ${ }^{10-12}$ but the mechanistic aspects of this intergenerational transmission of information, the so-called 'epigenetic memory', remained unclear. In particular, how is the balance between the acetylated and unmodified forms inherited and preserved. Here, we show that cell type-specific RNAs are conduits of the accurate recycling of the epigenetic mark and thereby provide transgenerational design marks for regulation of the transcriptional activity of the respective genomic loci.

Widespread transcriptional activity across the mammalian genome results in the production of many functional noncoding RNAs (ncRNAs) ${ }^{13-18}$ that play critical roles in multiple biological processes $^{18-29}$. Such RNAs are essential components of the chromatin architecture ${ }^{30-32}$ that is shaped by epigenetic marks such as DNA methylation, histone modifications, nucleosome positioning, and the incorporation of histone variants into nucleosomes. Despite their well-documented role, few of these RNAs have been fully characterized and the majority still lack a functional classification.

The overall effect of these ncRNAs is to regulate cell-type specific gene expression at both the transcriptional and post-transcriptional level, including: (i) chromatin remodeling by recruiting complexes that lead to epigenetic changes (e.g., polycomb repressive complex (PRC2)-mediated transcriptional regulation ${ }^{33}$; (ii) transcriptional interference by directly interacting with ubiquitous or tissue specific transcription factors (e.g., dissociation of the pre-initiation complex through sequestration of the general transcription factor $\mathrm{IIB}^{34}$ or Sp1 in Myotonic Dystrophy cases ${ }^{24}$; or (iii) splicing interference by affecting the distribution of serine/arginine splicing factors ${ }^{35}$.

A targeted and global genomic approach is used here to demonstrate that the deposition and modification of the active versus inactive H2A.Z mark is mediated by locally induced cell-cycle specific RNAs, termed SPEARs (S Phase EArly RNAs).

\section{RESULTS}


Ebralidze A.K. et al. text and figure legends

\section{Expression of newly minted promoter RNAs correlates with that of neighboring genes}

Since the majority of active genes undergo replication in early $\mathrm{S}$ phase, which is followed by formation of the appropriate active chromatin ${ }^{36}$, we reasoned that transcripts generated in early $\mathrm{S}$ phase might control expression of the respective mRNAs by playing a role in reassembly of the chromatin. Furthermore, we hypothesized that the epigenetic balance between acetylated and unmodified forms of H2A.Z could be maintained by locally induced ncRNAs.

To establish the global occurrence of such newly minted RNAs, nascent S-phase transcripts were captured and sequenced (nasRNA-Seq). Synchronized human HL-60 cells were labeled with the ribonucleotide homolog 5-ethynyl uridine (EU) for one hour upon release into S phase. The collected RNAs were then biotinylated by click chemistry, isolated on streptavidin beads and deep-sequenced to produce nasRNA-Seq libraries ${ }^{37,38}$ (Extended Data Fig. 1a and Methods for details). Analysis of such libraries demonstrated a strong correlation of expression levels between nascent transcripts arising from gene promoter regions ("nascent promoter RNAs") and those of their linked genes (Fig. 1a). In particular, such correlations are more prominent for nascent transcripts arising from the promoter regions of highly expressed genes (Fig. 1a). Among the early expressing genes marked by the presence of early S-phase promoter RNAs we identified $c-M Y C$ (Extended Data Fig. 1b,c), the oncogene most frequently altered in cancers ${ }^{39}$, myeloid master regulator transcription factor PU.1 (ref. ${ }^{40}$ ) and proto-oncogene $M Y B$ (ref. ${ }^{41}$ ) (uninterrupted sequence segments are in Supplementary

Data \#2); a list of all gene loci expressing early promoter RNAs is in Supplementary Data \#1. The $c$ $M Y C$ early S-phase promoter transcripts were shown to be represented by $\sim 13$ copies in the nucleus of HL-60 cells (Extended Data Fig. 1d) and were mapped by primer extension and 5', 3'-RACE (Extended Data Fig. 1e,f).

\section{A fraction of early S-phase promoter RNAs interacts with H2A.Z, acH2A.Z and TIP60}

Given the close proximity of promoter RNAs to TSS and the growing body of literature showing 
Ebralidze A.K. et al. text and figure legends

that highly expressed genes are marked by the acetylated form of the variant histone H2A.Z

(acH2A.Z) (ref. ${ }^{5}$ ), the possibilities of a link between early S-phase promoter RNAs and

H2A.Z/acH2A.Z was investigated by: (i) Ribonucleoprotein (RNP) pull-down experiments followed by mass spectrometry; and (ii) RNA immunoprecipitations followed by RNA sequencing (RIP-Seq).

To design the RNA probes for RNP pull-down, the sequences of three early S-phase promoter RNAs, identified by nasRNA-Seq (Supplementary Data \#1) and derived from gene loci $c-M Y C$, PU.1, and $M Y B$, were verified by primer extension and 5', 3' RACE (Figures S1E and S1F). The approximately $500 \mathrm{nt}$ uninterrupted sequence segments (Supplementary Data \#2) were cloned under a T7 RNA polymerase promoter to enable expression of biotinylated sense and antisense early promoter RNAs probes for RNP pull-down experiments (Extended Data Fig. 2a). Recovered RNPs interacting with both the antisense and sense early S-phase promoter RNAs probes and a negative control (D-Biotin) were analyzed by mass spectrometry. Among peptides pulled down by the early promoter RNAs probes were several corresponding to H2A.FZ/ H2A.FV (Supplementary Data \#3). It was not possible to distinguish the very homologous forms of H2A.FZ vs. H2A.FV, and no peptides containing acetylation sites were detected, due to the relatively low fragment coverage. Other peptides pulled down by the early promoter RNA probes corresponded to histones $\mathrm{H} 2 \mathrm{~A}, \mathrm{H} 2 \mathrm{~B}$, H3, H4 and H1. No peptides corresponding to H2A/H2A.Z or other histones were detected by the negative control probe (Supplementary Data \#3).

It is possible that early S-phase promoter RNAs exert their function through direct interaction with H2A.Z and the histone acetyl-transferase (HAT) TIP60, the enzyme reported to acetylate H2A.Z ${ }^{9,42}$. To directly test the interaction of early S-phase promoter RNAs with H2A.Z, acH2A.Z, and TIP60, RIP-Seq experiments were performed using antibodies to H2A.Z (i.e. recognizing all forms of the histone), to acetylated H2A.Z (acH2A.Z), and to TIP60 (Extended Data Fig. 2b). A significant overlap of RIP-Seq peaks was observed from all three immunoprecipitations and considerable colocalization of the three data sets was detected in the regions around the mRNA TSSs. In particular, a 
Ebralidze A.K. et al. text and figure legends

significant enrichment was observed of both sense and antisense transcripts in the $2 \mathrm{~kb}$ window upstream of TSSs (total number of early S-phase nascent promoter RNAs: 4689; 2,411 and 2,278 transcripts on the sense and antisense strands, respectively; Fig. 1c,d). At this stage, all early promoter RNAs which did not have detectable expression above background in the nasRNA-Seq library were discarded, which restricted the list of early S-phase nascent promoter RNAs to 3,560 (1,810 and 1,750 transcripts on the sense and antisense strands, respectively) (Fig. 1e). To assess the functional impact of the interaction of the early S-phase nascent promoter RNAs with TIP60, H2A.Z, and acH2A.Z, the identified 3560 early S-phase nascent promoter RNAs were aligned with the acH2A.Z ChIP-Seq library. To ensure consistency and stringency, only gene loci with a detectable acH2A.Z ChIP signal in the $2 \mathrm{~kb}$ upstream window of the TSS were accepted (Fig. 1f). This identified 2363 promoter RNAs (1209 sense and 1154 antisense transcripts) which are expressed in the first hour of S phase and which overlap with the signals of all four data sets: the three RIP-Seq libraries (antibodies to H2A.Z, TIP60, and acH2A.Z) and the acH2A.Z ChIP-Seq library. The colocation of acH2A.Z ChIP-Seq signals in the same genomic region as the signals from the three independent RIP-Seq experiments suggests a functional role of the early S-phase nascent promoter RNAs in positioning the acetylated H2A.Z marks. We termed these 2,363 early S-phase nascent promoter RNAs SPEARs (S Phase EArly RNAs). Comparison of the H2A.Z and acH2A.Z ChIP-Seq data sets implies that it is the acetylated form of H2A.Z that is largely bound to the SPEARs (Fig. 1g). Taken together, the histone immuno-precipitations and the RIP experiments indicate: i) a triple interaction of SPEARs with H2A.Z, acH2A.Z, and with TIP60 in the $1 \mathrm{~kb}$ upstream of the TSS (Extended Data Fig. 2c); and ii) a correlation of SPEARs expression level with that of the linked genes.

In summary, identification of 2,363 SPEARs linked to expressed genes, direct correlation of SPEARs expression levels with those of the corresponding genes, and, importantly, with the occupancy of acH2A.Z within the respective loci (Fig. 1f-h), implies a global involvement of 
Ebralidze A.K. et al. text and figure legends

SPEARs in cooperation with TIP60 and H2A.Z/acH2A.Z in establishing an active expression mode at the corresponding genes. This warranted further examination.

\section{SPEARs carry common binding motifs}

To further test the SPEARs-H2A.Z/TIP60 relationship, we performed motif discovery analysis on the SPEARs (see Methods for details). Among the twenty-five identified motif candidates, three motifs (\#\#3, 5, and 9; Supplementary Data \#4) exhibited the most significant enrichment among the SPEARs-regulated gene loci. Importantly, no similar motifs were found within the largest classes of nuclear RNAs, i.e. transcripts arising from SINEs and LINEs, nor from ribosomal RNA genes (see Methods for details). Among the top three candidates, motif 9 (corresponding to the RNA oligonucleotide RM9A; Fig. 2a and Extended Data Fig. 3a) was ranked as the strongest motif enriched in the $c$-MYC SPEARs sequence. This motif was enriched in acH2A.Z and TIP60 RIP-Seq overlapping peaks (13\%), located near the peak centers (Fig. 2b).

RNA electrophoresis mobility shift assays (REMSAs) using the RM9A motif demonstrated that it is able to form RNPs with H2A.Z and with TIP60 in vitro (Fig. 2c, lane 3; and Fig.2d, lane 4). A shift in migration was also observed by incubation of RM9A with synthetic peptides corresponding to the N-terminal sequences of H2A.Z, both unmodified and acetylated at lysine 7 (K7) (Fig. 2c, right panel). Acetylation of the H2A.Z tail peptide did not abrogate the binding (Fig. 2c, lanes12, 13 - acetylated peptide, and lanes 8,9-not acetylated peptide), indicating that simple charge-charge interactions are not responsible for SPEARs-H2A.Z or SPEARs-acH2A.Z complexes. Interestingly, the presence of a predicted stem-loop-like structure (RNAfold ${ }^{43,44}$; Fig. 2e) seems to be required for RM9A binding to H2A.Z and TIP60 (Fig. 2c,d), but unlike the previously identified DiRs (DNMT1interacting RNAs $)^{45}$, the secondary structures are not the only requirement for RNP formation. Indeed, mutation of RM9A so as to prevent the predicted stem-loop structure reduced but did not 
Ebralidze A.K. et al. text and figure legends

totally abrogate the formation of the RNA-TIP60 complexes (Fig. 2d, lanes 4-5). Importantly, unrelated oligonucleotides (UR2 and UR3) lacking the common binding motif did not form comparable RNP complexes with TIP60/H2A.Z (Extended Data Fig. 3), indicating the involvement of both primary and secondary structure elements in the recognition of TIP60/H2A.Z by RM9A.

RNAs might be important for binding of remodeling complexes to chromatin, as found for the polycomb repressive complex $2(\mathrm{PRC} 2)$ and Tip60-p400 ${ }^{13,46-48}$. To test if SPEARs facilitate binding of TIP60 to the chromatin, we compared TIP60 binding to the RNA (single-stranded RM9A) and DNA (double-stranded DM9A) having the same primary sequence. The REMSA/EMSA gels indicate very strong TIP60 binding to the ssRM9A and weaker binding to the dsDM9A (Fig. 2d, lane $4 v s$. lane 6).

In conclusion, a specific interaction is seen between the RM9A region of the c-MYC SPEAR and the histone acetyltransferase TIP60 and histone H2A.Z, in particular its modified form acH2A.Z.

\section{SPEARs are involved in H2A.Z acetylation and exchange}

To directly test if SPEARs are required for the deposition of acH2A.Z at the TSS of their corresponding gene, transcription was pharmacologically modified and followed by chromatin immunoprecipitation/sequencing (ChIP-PCR and ChIP-Seq) using H2A.Z and acH2A.Z antibodies (outlined on Extended Data Fig. 4a). Two transcription inhibitors, Actinomycin D (ActD; a RNA Polymerase I, II, and III inhibitor) and 5,6-Dichlorobenzimidazole 1- $\beta$-D-ribofuranoside (DRB; a RNA Polymerase II Inhibitor), were used at concentrations sufficient to interfere with the transcripts produced by both RNA Polymerases II and III. The rationale behind the use of ActD and DRB is: (i) to globally assess the effect of changes in SPEARs expression levels over a short time frame;

(ii) to compare effects resulting from the two different pathways triggered by ActD and DRB; and (iii) to take advantage of the reversibility of DRB treatment. Cells were synchronized with a double thymidine block and released into $\mathrm{S}$ phase with ActD/DRB added (Extended Data Fig. 4a), treated 
Ebralidze A.K. et al. text and figure legends

with the drugs for two hours, an interval during which the overall levels of H2A.Z and TIP60

proteins were not affected (Extended Data Fig. 4b), while the global RNA levels of both the coding genes and the SPEARs were significantly altered (Fig. 3a). Relative changes were then investigated in the distributions of unmodified H2A.Z and acH2A.Z in the vicinity of the TSS of the SPEARslinked genes, in response to DRB- and ActD-induced changes in SPEARs expression levels. The enrichments of ChIP-Seq signals for H2A.Z and acH2A.Z in the region adjacent to the TSSs of the 2363 SPEARs-linked genes following drug treatment were compared with their occupancy in mocktreated (DMSO) cells. We observed substantial reductions in acH2A.Z enrichment at the TSS of samples treated with either DRB or ActD (Fig. 3b, right panels), in contrast to only modest changes in the occupancy levels of unmodified H2A.Z (Fig. 3b, left panels). In cells treated with DRB, 26\% of identified SPEARs loci showed a drop in acH2A.Z occupancy, while only $6 \%$ of such loci showed a similar trend in the H2A.Z signal (Fig. 3b, upper panels). ActD-treated cells showed a more pronounced effect, with $42 \%$ of SPEARs loci showing reduced acH2A.Z signal as compared with $4 \%$ of such loci showing decreased occupancy of H2A.Z (Fig. 3b, bottom panels). Thus, Fig. 3b demonstrates that loci with suppressed expression of SPEARs showed diminished enrichment in acH2A.Z, implying that SPEARs are involved in the precise placement of this epigenetic mark. The observation of a 10-fold greater number of acH2A.Z-affected TSS in drug-treated samples as compared to H2A.Z, indicates the involvement of SPEARs in the maintenance of normal levels of H2A.Z acetylation at TSS, i.e. to the generation of the active epigenetic mark acH2A.Z. The upper panel of Fig. 3c depicts snapshots of the $c-M Y C$ locus for which the SPEARs are repressed by ActD and DRB treatment (results of qRT PCR are shown in Extended Data Fig. 4c, left panel), resulting in a decreased intensity of acH2A.Z peaks. In detail, for the $c-M Y C$ the ratios of control (DMSO) to drug treated acH2A.Z peak intensities were $[\mathrm{DMSO} / \mathrm{DRB} / \mathrm{ActD}]^{c-M Y C}=1 / 0.45 / 0.39$. These ChIP-Seq data were confirmed by quantitative ChIP-qPCR for several amplicons covering the $c$-MYC locus (Extended Data Fig. 4d). By contrast, loci escaping the down-regulating effects of the drugs on their 
Ebralidze A.K. et al. text and figure legends

SPEARs (the "DRB paradox"49) exhibit an acH2A.Z intensity ratio in simple correlation with the change in SPEARs levels. For example, the MYB locus gives rise to SPEARs positively affected by ActD and DRB (Extended Data Fig. 4c, right panel), and exhibits an increase in the intensity of the acH2A.Z peaks, with no significant changes in the intensity of the unmodified H2A.Z peaks (Fig. 3c, bottom panel). For $M Y B$, the ratios of control (DMSO) to drug treated acH2A.Z peak intensities are $[\mathrm{DMSO} / \mathrm{DRB} / \mathrm{ActD}]^{M Y B}=1 / 1.52 / 1.60$, which correlates with the increases in its SPEARs levels (Extended Data Fig. 4c, right panel). Thus, loci at which expression of SPEARs are positively affected by ActD/DRB illustrate how an increase in SPEARs levels leads to a corresponding rise in the intensity of the acH2A.Z peaks.

Nascent chromatin immunoprecipitation (nasChIP-PCR ${ }^{50-52}$ ) is particularly useful for tracking histone deposition and was therefore used with the antibodies to H2A.Z, acH2A.Z and TIP60. Cells were synchronized with a double thymidine block, released into S phase and treated with DRB for 2 hours. The medium was supplemented with the EdU DNA analog to enable collection of nascent chromatin DNA (Extended Data Fig. 4a). It was observed that when transcription was inhibited with DRB, the $c-M Y C$ locus showed diminished enrichment in H2A.Z, acH2A.Z and TIP60 (Extended Data Fig. 4e, three upper panels). This demonstrates a link between suppressed $c-M Y C S P E A R s$ expression and reduced levels of the replacement histone H2A.Z on the nascent chromatin and an even greater reduction of the acetylated acH2A.Z at the TSS of the c-MYC locus. Similar results were obtained for the PU.1 gene locus (Extended Data Fig. 4e, three bottom panels).

Taken together, the negative effects of ActD/DRB - a global "loss-of-function", exemplified by $c-M Y C$, - and their positive effects, a global "gain-of-function", exemplified by MYB - monitored by ChIP-Seq and nasChIP-PCR experiments, define the role of SPEARs transcripts in deposition of the replacement histone H2A.Z. The more robust drop in the acetylation level of H2A.Z as compared to the more modest decrease in the unmodified form, as well as loss of TIP60 at the same sites close to 
Ebralidze A.K. et al. text and figure legends

the TSSs of the genes, all point to the role of SPEARs transcription in mediating the acetylation of H2A.Z.

In complementary gene-specific experiments, we then tested whether RNAi-mediated downregulation and dCas9-VP64-mediated upregulation of specific SPEARs followed by ChIP-Seq and ChIP-qPCR analyses leads to a corresponding reduction or increase of H2A.Z and acH2A.Z levels at the TSS of targeted loci and in reduced or increased expression of the corresponding gene. Knockdown of the $c-M Y C$ SPEARs ( 75\%; Fig. 3d, left panel) was associated with a significant decrease of acH2A.Z levels at the TSS of the $c-M Y C$ gene (Fig. 3d, middle panel), followed by the corresponding decrease of $c-M Y C$ mRNA expression of similar magnitude ( 70\%; Fig. 3d, right panel). The results of the RNAi/ChIP knockdown experiments demonstrate that reduced expression of the $c$-MYC SPEARs is linked to lower levels of H2A.Z acetylation at the $c$-MYC TSS, which accords with the initial hypothesis of direct causality between the expression of SPEARs and the deposition of the activating histone mark, leading to transcriptional activation of the adjacent gene.

Additionally, we performed a "gain-of-function" test using the dCas9-VP64 gene activation system (outlined on Extended Data Fig. 4f). Increasing levels of $c$-MYC SPEARs expression (Fig. 3e, left panel) were associated with a significant increase of acH2A.Z levels at the TSS of the $c-M Y C$ gene (Fig. 3e, middle panel) accompanied by a $~ 2$-fold increase in $c$-MYC gene expression (Fig. 3e, right panel).

Overall, downregulation of SPEARs using independent pharmacological and RNAi-induced methods leads to a substantial reduction in the activating epigenetic mark acH2A.Z. Loss of the acetylated form of $\mathrm{H} 2 \mathrm{~A} . \mathrm{Z}$ from chromatin in the vicinity of active TSS implies a reduction of its activity, i.e. reduced expression of the corresponding gene (Fig. 3d, right panel). On the other hand, upregulation of SPEARs leads to a substantial induction of the activating epigenetic mark acH2A.Z and, consequently, to elevated expression of the corresponding gene (Fig. 3e, right panel). 
Ebralidze A.K. et al. text and figure legends

\section{SPEARs regulate the expression of the corresponding mRNA via TIP60 recruitment and}

\section{acH2A.Z deposition}

To demonstrate that the SPEARs-mediated regulation of their corresponding mRNA is executed through the TIP60/acH2A.Z pathway, the effects of TIP60 inhibition on the c-MYC locus were tested. Two TIP60/HAT inhibitors, TH1834 ${ }^{53}$ and MG-149 ${ }^{54}$, were used in HL-60 cells to reduce the total level of H2A.Z acetylation (Extended Data Fig. 5a). This led to significant downregulation of mRNAs, in contrast to almost unchanged levels of SPEARs transcripts (Fig. 4a). To examine the direct regulation of $c-M Y C$ expression by TIP60 and acH2A.Z interacting with the SPEARs, we monitored the regulating effect of nascent SPEARs on the level of nascent mRNA expression in the presence or absence of the two TIP60 inhibitors, taking advantage of the reversibility of the DRB transcriptional inhibitor (ref. ${ }^{55}$. The rationale was to analyze only transcripts re-appearing after release from the transcriptional block whilst the HAT is inhibited. The short time of inhibitor treatment should rule out secondary effects of HAT inhibition.

Cells were synchronized with a double thymidine block and released into S Phase with DMSO/DRB added. After 2 hours (during which H2A.Z levels remained unaffected, but SPEARs and mRNA levels drop (see Extended Data Fig. 5a,b), cells were washed and then incubated for a further 2 hours with or without the TIP60 inhibitors. This interval is sufficient for the levels of both the $c-M Y C$ mRNAs and its SPEARs to be restored to the levels when the TIP60 inhibitors are absent (results shown in Extended Data Fig. 5b). The medium was supplemented with the EU RNA and EdU DNA analogs to enable collection of nascent RNAs and newly formed chromatin that had escaped the drug-induced inhibition of transcription or acetylation. After 2 hours with the TIP60 inhibitors present (MG-149 at $200 \mu \mathrm{M}$ and TH1834 at $500 \mu \mathrm{M}$ ), during which time the levels of acH2A.Z dropped (see Extended Data Fig. 5a), cells were crosslinked and subjected to nasChIP-PCR and nascent RNA expression analyses (nas-qRT-PCR) (outlined on Fig. 4b). Collected RNAs were 
Ebralidze A.K. et al. text and figure legends

biotinylated by click chemistry, isolated on streptavidin beads and analyzed by nas-qRT-PCR (see Methods for details and Extended Data Fig. 1a, bottom panel). The results in Fig. 4c show that the correlation between expression of the SPEARs and the mRNA no longer holds when TIP60 activity is inhibited, i.e. the restored levels of the SPEARs are incapable of rescuing the expression of $c-M Y C$ mRNA to the level defined by the reversal of DRB repression. These data suggest that the function of SPEARs in regulating $c-M Y C$ mRNA expression is impeded by inhibition of the HAT activity of TIP60.

To further check that SPEARs realize their $c-M Y C$ regulatory function directly through the TIP60/acH2A.Z pathway, we assessed the differences in chromatin occupancy of H2A.Z, acH2A.Z and TIP60 after inhibition of transcription ("DRB"), reversal of inhibition ("DRB REV”), and reversal of inhibition in the presence of the two TIP60 inhibitors, using nasChIP-qPCR. In particular, it was important to check whether the drop in $c-M Y C$ expression after treating the cells with DRB and TIP60 inhibitors is correlated with diminished enrichment in TIP60, acH2A.Z, or H2A.Z. Nascent DNA was isolated from the chromatin immuno-precipitated with antibodies to H2A.Z, acH2A.Z, or TIP60, biotinylated by click chemistry, isolated on streptavidin beads (Methods) and finally analyzed by qPCR at amplicons corresponding to maximum enrichment within the $c-M Y C$ locus (see Fig. 3e). By analyzing the nascent RNAs and chromatin, we examined only the immediate changes in $c-M Y C$ expression and TIP60, acH2A.Z, and H2A.Z occupancy imposed by the inhibition of transcription and/or the reduced acetylation of histones. The middle and right panels of Fig. $4 \mathrm{~d}$ demonstrate that the $c$-MYC locus with suppressed expression of its SPEARs shows diminished enrichment of both acH2A.Z and TIP60, and the reversal of the transcription inhibition led to the restoration of both enrichments. This indicates that the SPEARs are involved in the recruitment of TIP60 and in the proper placement of the acetylation mark. When the two HAT inhibitors are present, the reversal of transcriptional inhibition can only restore the enrichment levels of unmodified H2A.Z and of TIP60 (Fig. 4d, left and right panels) but not the levels of acH2A.Z (Fig. 4d, middle 
Ebralidze A.K. et al. text and figure legends

panel). In contrast, no enrichments of H2A.Z, acH2A.Z, or TIP60 were detected within the Gene

Desert locus $^{56}$ (Extended Data Fig. 5c). Given that local levels (i.e. in the vicinity of the $c$-MYC locus

TSS; Fig. 4d, left panel) as well as overall levels of H2A.Z were unchanged throughout the

experiment (Extended Data Fig. 5a), these results establish the leading role of the TIP60/acH2A.Z

pathway in $c-M Y C S P E A R s$-mediated regulation of its corresponding gene, $c-M Y C$. Similar results

were obtained for the PU.1 locus (Extended Data Fig. 5d,e ) and with another HAT inhibitor - NU

9056 (which at high concentration inhibits acetylation of H2A.Z) (Extended Data Fig. 5f-h). As with

HAT inhibitors MG149 and TH1834 (Fig. 4d, middle panel), we observed loss of promoter

occupancy by acH2A.Z after treatment with the HAT inhibitor NU 9056 in cells pre-treated with

DRB (“DRB/NU 9056”) (Extended Data Fig. 5h).

Collectively our results suggest that the TIP60/acH2A.Z pathway is a general mechanism for SPEARs-mediated regulation of the corresponding adjacent coding genes.

\section{SPEARs regulate recycling of the epigenetic mark - acH2A.Z}

To demonstrate the role of SPEARs in the recycling of the acH2A.Z mark, we performed the experiments outlined in Fig. 5a. Cells were cultured for six days in medium supplemented with "heavy" ${ }^{15} \mathrm{~N}_{2}{ }^{13} \mathrm{C}_{2}$-Lysine and ${ }^{13} \mathrm{C}_{6}$-D-glucose and then synchronized with a double thymidine block. After washing out "heavy" lysine and glucose, cells were released into S phase in the presence of HAT inhibitor or DMSO (mock treatment). The rationale of the experiment was to distinguish histones deposited in the chromatin before entering S phase from those deposited after this point. Thus, histones containing "heavy" lysines and their acetylated forms carrying "heavy" acetyl groups are "old", whilst "new" histones and their acetylated forms generated during early S phase should contain "light" lysines and "light" acetyl groups. The medium was supplemented with the EdU DNA analogs to enable collection of newly formed (nascent) chromatin. The HAT inhibitor NU 9056 was added at the start of S-phase and the efficiency of inhibition was demonstrated by HAT assays on the 
Ebralidze A.K. et al. text and figure legends

collected cells (Extended Data Fig. 6a) showing efficient suppression of H2A.Z acetylation. This experiment enabled investigation of whether "old" histones with "old" modifications are preserved and transmitted through the generational border. At 4 hours after release from synchrony, cells were collected and subjected to gene expression and Western Blot analyses, HAT assay, ChIP-PCR, nasChIP-PCR, and mass spectrometry. As with TH1834 and MG148 inhibition (Fig. 5a,b), treatment with NU 9056 resulted in a drop in mRNA but not in SPEARs expression (Fig. 5b) and levels of H2A.Z remained unaffected but acH2A.Z levels dropped (Fig. 5c).

To verify that the $c-M Y C$ locus indeed undergoes replication and chromatin re-assembly in the early part of S phase, nasChIP-PCR was performed (with antibodies to acH2A.Z) and a significant enrichment of acH2A.Z-occupied nascent $c$-MYC locus chromatin was observed (Fig. 5d).

The tests of experimental conditions being satisfactory (Extended Data Fig. 6a and Supplementary Data \#5), ChIP-PCR analyses of acH2A.Z occupancy at the target promoters was performed. Strikingly, analysis of promoter occupancy by acH2A.Z in HL-60 cells after even longer treatment in NU 9056 showed levels of acH2A.Z to be diminished by only one half in comparison to the mock treatment (Fig. 5e, right panel). Two points need emphasis: (i) when acetylation of all newly synthesized histones is strongly inhibited, persistent promoter occupancy by the acetylated forms of H2A.Z can occur only through the recycling of the previously acetylated ("old") histones; and (ii) this recycling process is interrupted by the inhibition of transcription, particularly with the abrogation of SPEARs expression (see Fig. 4 and 5).

To unequivocally demonstrate that the "recycling" of acetylated forms of histones indeed represents "old" histones, we performed mass spectrometry analyses of the immunoprecipitates from cells subjected to HAT inhibitor for 4 hours" (Fig. 5a), in which all "new" acetylation was suppressed. The samples were digested with chymotrypsin and analyzed on an Orbitrap Elite mass spectrometer. The Proteome Discoverer 2.4 search engine analyzed the raw data. Searches were performed against the H2A.Z protein sequence and common contaminants and all results filtered to 1 
Ebralidze A.K. et al. text and figure legends

$\%$ FDR (false discover rate). The analysis focused on the N-terminal H2A.Z peptide Ac-

AGGKAGKDSGKAKTK (Extended Data Fig. 6b) with variable acetylation of lysines K4, K7, and K11 and showed a high level of "heavy" lysines and the presence of heavy acetyl groups in this Nterminal peptide. Analysis of isotopic distributions showed that among all N-terminal peptides carrying two acetyl groups, the content of heavy lysines was $\sim 70 \%$ and of heavy acetyl groups was $\sim 20 \%$. In peptides carrying three acetyl groups the content of heavy lysines was $\sim 55 \%$ and of heavy acetyl groups $\sim 30 \%$. Extended Data Fig. 6c shows examples of the peptides detected. Fig. $5 f$ demonstrates an increase of heavy acetyl groups in the "HATs inhibitor 4 hours" sample (Left panel; $\sim 70 \%$ ) as compared to their natural distribution (Right panel; $\sim 38 \%$ ). These analyses show that some old H2A.Z histones carrying heavy acetyl groups are preserved in the early stages of S phase and that recycling of old acH2A.Z is affected by brief suppression of transcription in the first hours of $\mathrm{S}$ phase.

\section{Discussion}

The roles of ncRNAs in regulating deposition and recycling of acH2A.Z at gene promoters has been investigated. Transcription of SPEARs that control the acetylation of nearby H2A.Z could be regarded as a mechanism whereby information encoded in the genome becomes expressed as 'epigenetic' information. Locally induced SPEARs bind to the replacement histone H2A.Z and to a nuclear factor, the histone acetyl transferase TIP60, leading to deposition/acetylation of H2A.Z, a process that represents recycling of the mark across generations. SPEARs transcripts guide deposition/acetylation of this replacement histone through RNP formation with TIP60/H2AZ. The function of these transcripts depends on a combination of their expression levels, primary and secondary structures, and physical co-compartmentalization of RNAs, their parental loci, and their protein partners. SPEARs databases can provide a valuable resource for studying the dynamics of 
Ebralidze A.K. et al. text and figure legends

RNP assembly and their role in the formation of active/inactive chromatin. This mechanistic model is depicted in Figure 6.

The demonstrated link between SPEARs and the activated form of the replacement histone H2A.Z raises the possibility that other long ncRNAs (lncRNAs) play a role in the recruitment of chromatin modifiers that deposit activating or repressive epigenetic marks. Although more detail is still needed, such lncRNAs may act not only as signaling molecules but also as the scaffolds around which chromatin writer/eraser complexes are assembled. This notion is supported by an observation in Drosophila, in which the deposition of the replacement histone H3.3 occurs within transcriptionally active loci and is not replication-coupled ${ }^{57}$.

In summary, the discovery of SPEARs reveals a novel mechanism for the biosynthesis and maintenance of a major epigenetic mark. Taken together with the study of identified $D i R s^{45}$, the present investigations demonstrate that epigenetics and genetics are intrinsically linked, and such lncRNAs modulate distinct epigenetic marks by using site-specific targeting molecules. Indeed, epigenetic modulating agents are already employed in the clinic ${ }^{58,59}$, but assessment of their function is difficult, their efficacy being compromised by lack of specificity. This research provides a new tool to overcome these drawbacks by using the specificity of RNA molecules to drive deposition and selective modification of histones. This study indicates the need for further investigations uncovering how epigenetic information is encoded in the genome and regulated under normal conditions and in disease states.

\section{METHODS}

Detailed methods are provided in the online version of this paper and include the following:

\section{- KEY RESOURCES}

- CONTACT FOR REAGENT AND RESOURCE SHARING

- EXPERIMENTAL MODEL 
Ebralidze A.K. et al. text and figure legends

Mammalian Cell Culture

- METHOD DETAILS

RNA isolation;

qRT-PCR;

Nascent RNA/DNA capture;

Primer extension and 5'/3' RACE;

Double Thymidine block (early S-phase block);

DRB and Actinomycin D treatments;

Down-regulation of $c-M Y C S P E A R s$;

Ribonucleoprotein (RNP) fractionation;

Western Blotting Analysis;

Tandem Mass Spectrometry (LC-MS/MS);

Nuclear Chromatin (ChIP) and RNA immunoprecipitation (nRIP);

RNA electrophoretic gel mobility shift assays (REMSAs).

- QUANTIFICATION AND STATISTICAL ANALYSIS

ChIP- Sequencing Analyses;

Comparison of the enrichment of H2A.Z and acH2A.Z ChIP-Seq signal surrounding gene TSS loci (+/- 2kb);

RIP-Sequencing Analyses;

RNA-Sequencing Analyses;

Motif Discovery;

LC-MS/MS Analyses.

- DATA AND SOFTWARE AVAILABILITY 
Ebralidze A.K. et al. text and figure legends

Sequencing Data are available on the gene omnibus database under the accession ID number: GSE165526; enter token: slehymyczncvfqd. List of all data sets is in Supplementary Data \#6. Mass Spectrometry data will be deposited to the ProteomeXChange Consortium via PRIDE upon acceptance of the manuscript. Private partial submission with MASSIVE:

https://massive.ucsd.edu/ProteoSAFe/dataset.jsp?task=38a5eae58dd247f5b3c42f2be181e692;

Password: Alex_Acetyl

\section{Figure Legends}

Fig. 1. Characterization of the promoter RNAs induced in the first hour of $S$ phase.

a, Genome-wide analysis of the nascent nuclear RNAs (nasRNAs). Correlation between the nascent promoter RNA level and the expression of their respective coding genes. Pearson's correlation score is 0.54 , with an $\mathrm{R}^{2}$ value of 0.3 , and p-value $<0.0001$. b, Ratio of Promoter to Exon expression of Sphase nascent nuclear transcripts and of S-phase total nuclear transcripts, both in the first hour. Pvalue < 0.0001. c-h, SPEARs interactions with H2A.Z, acH2A.Z and TIP60. c, Read coverage plots for sense (forward) and antisense (reverse) strands for the region $-2 \mathrm{~kb}$ to $+2 \mathrm{~kb}$ surrounding transcriptional start sites (TSS). Note enhanced signal in upstream $2 \mathrm{~kb}$ region compared to downstream $2 \mathrm{~kb}$ region for all transcripts on both strands. d, Left Panel (Sense strand) a total of 40,326 RIP peaks overlapped the H2A.Z (green), TIP60 (orange) and acH2A.Z (purple) RIPs using a merge window of $1 \mathrm{~kb}$. From the 40,326 overlapping peaks those having a center located in the range $-2 \mathrm{~kb}-\mathrm{TSS}-+1 \mathrm{~kb}$ were selected, resulting in 2,411 being retained. Right Panel (Anti-Sense strand) a total of 39,695 RIP peaks overlapped the H2A.Z (green), TIP60 (orange) and acH2A.Z (purple) RIPs using a merge window of $1 \mathrm{~kb}$. From the 39,695 overlapping peaks those having a center located in the range $-2 \mathrm{~kb}-\mathrm{TSS}-+1 \mathrm{~kb}$ were selected, resulting in 2,278 being retained. Altogether, 4,689 
Ebralidze A.K. et al. text and figure legends

promoter RNAs were identified from a total of 4,351 genes across both strands. e, RIP-Seq signals: read coverage plot for the region $-2 \mathrm{~kb}$ to $+2 \mathrm{~kb}$ surrounding TSSs. A significant enrichment of transcripts is seen in the upstream $2 \mathrm{~kb}$ region. $\mathbf{f}$, Total acH2A.Z ChIP-Seq signals in the same region as RIP-Seq signals in Panel E, highlighting the increase in promoter signal at the same position as the S phase nascent promoter RNAs. g, H2A.Z and acH2A.Z ChIP-Seq signals overlapped just with confirmed SPEARs. h, Upper Panel - A normalized genomic coverage plot showing identified SPEARs in the region of the $c-M Y C$ TSS. The normalized coverage signals for each sample are overlaid to show good co-mapping of signals for H2A.Z (green), TIP60 (orange) and acH2A.Z (purple). The results of the qRT PCR (using the indicated amplicon) are shown in Figure S2CD. Bottom Panel: the acH2A.Z ChIP-Seq signals in the same region of the $c$ - $M Y C$ gene, highlighting increased signal at the same positions as the $c-M Y C$ SPEARs.

\section{Fig. 2. Identification of common binding motifs in $S P E A R s$.}

a, The sequence of the common binding motif "9" (RNA oligonucleotide RM9A) and the unrelated RNA oligonucleotides (UR) within the c-MYC SPEARs, along with the corresponding motif "9" DNA duplexes (DM9A) are indicated. b, Distribution of the RM9A motif in acH2A.Z and TIP60 RIP-Seq overlapping peak regions. Significance and percent enrichment indicated. c, Left panel: REMSA gel for $c$-MYC SPEARs carrying the RM9A binding motif forming specific complexes with full-length histone H2A.Z. Right panel: similar REMSA using non-acetylated and acetylated peptides from the $\mathrm{N}$ terminal sequence of H2A.Z: ( $\mathrm{NH}_{2}$-AGGKAGK(Ac)DSGKAKTKAVSRS$\mathrm{COOH})$. d, REMSA gel for $c-M Y C$ SPEARs carrying the RM9A binding motif and forming specific complexes with TIP60 protein (lane 4) The mutated RNA and DNA motifs do not form complexes (lanes 5 and 6). Both RNA and DNA probes were used at the same molar concentration (0.5 nM), which corresponds to double the counts/minute for the double-stranded DNA duplexes relative to the single-stranded RNA oligonucleotides. 
Ebralidze A.K. et al. text and figure legends

\section{Fig. 3. Global and targeted up- and down-regulation of SPEARs expression leads to corresponding changes in occupancy of acH2A.Z at the TSSs of linked genes.}

a, Left Panel - Scatterplot of the global inverse hyperbolic sine (asinh) transformed counts-permillion (CPM) normalized Nuclear nascent RNA-Seq expression vs the asinh transformed acH2A.Z ChIP-Seq promoter signal count for DMSO (red), DRB (green), and ActD (blue) treated samples. Right Panel - Scatterplot of the confirmed SPEARs asinh transformed counts-per-million (CPM) normalized nuclear nascent RNA-Seq expression vs the asinh transformed acH2A.Z ChIP-Seq promoter signal count for DMSO (red), DRB (green) and ActD (blue) treated samples. Consistent with previous reports, a subset of genes show the "DRB paradox" in which their expression increases post-treatment. b, Density plots showing total promoter signal for DRB and ActD treatment vs DMSO for both H2A.Z and acH2A.Z ChIP-Seq samples. Top left panel: DRB treatment showed decreased H2A.Z promoter signal in $6 \%$ of genes. Bottom left panel, ActD treatment showed decreased H2A.Z promoter signal in $4 \%$ of genes. Top right panel: DRB treatment showed decreased acH2A.Z promoter signal in $26 \%$ of genes. Bottom right panel: ActD treatment showed decreased acH2A.Z promoter signal in $42 \%$ of genes. c, Snapshots of the individual loci, $c-M Y C$ and $M Y B$, for which the SPEARs are repressed or induced, respectively, by ActD and DRB treatment (results of qRT PCR are shown in Extended Data Fig. 4c), resulting in corresponding decrease or increase in the intensity of acH2A.Z peaks. d, siRNA-induced downregulation of $c$-MYC SPEARs ( 75\%; left panel) leads: i) to diminished acH2A.Z peaks (middle panel); and ii) to downregulation of $c-M Y C$ mRNA ( 70\%; right panel). qRT-PCR, bars indicate mean \pm s.d. e, CRISPR/dCas9-VP64 gene activation system-based upregulation of the $c M Y C$ SPEARs ( 3-fold; left panel) leads: i) to increased acH2A.Z peaks (middle panel; amplicon located $652 \mathrm{nt}$ to $345 \mathrm{nt}$ from $c$-MYC TSS); and ii) to upregulation of $c-M Y C$ mRNA ( $70 \%$; right panel). The position of tested Guide RNAs is shown in Extended Data Fig. 4f. qRT-PCR, bars indicate mean \pm s.d. 
Ebralidze A.K. et al. text and figure legends

Fig. 4. SPEARs regulate the expression of their linked mRNA via a TIP60/acH2A.Z pathway. a, Response of total $c-M Y C$ mRNA and SPEARs to TIP60/HAT inhibitors. $C-M Y C$ mRNAs are downregulated by MG149 and TH1834, but these inhibitors do not affect $c$-MYC SPEARs. HL-60 cells were released from double thymidine block and treated for 2 hours with MG149 (200 $\mu \mathrm{M})$ and TH1834 (500 $\mu \mathrm{M})$; control (mock) treatments were supplemented with DMSO $(0.05 \%)$ or water, respectively. qRT-PCR and strand-specific qRT-PCR were used to quantitate SPEARs and mRNA. Bars indicate mean \pm s.d. b, Experimental design: HL-60 cells were released into S Phase and treated with DMSO/DRB for 2 hrs (as described for Panel A), then washed and incubated for another 2 hrs with or without TIP60 inhibitors. The medium was supplemented with the EU RNA and EdU DNA analogs to enable collection of nascent RNAs and DNAs. Chromatin was collected for ChIP assays with antibodies to H2A.Z, acH2A.Z, TIP60, and IgG. Nascent DNAs and RNAs were isolated from the immunoprecipitated chromatin and from total RNA, respectively (see Methods for details; Extended Data Fig. 1a) and analyzed by qPCR and strand-specific qRT-PCR, respectively. c, Different response of nascent $c-M Y C$ SPEARs and $c-M Y C$ mRNA to TIP60/HAT inhibitors: $c-M Y C$ mRNAs are downregulated but $c-M Y C$ SPEARs are not affected. Quantitation was performed by qRT-PCR (for mRNA) and strand-specific qRT-PCR (for SPEARs): bars indicate mean \pm s.d. d, Nascent ChIP-qPCRs for the $c-M Y C$ locus using antibodies to: H2A.Z, acH2A.Z, and TIP60 with an IgG control (amplicon located $652 \mathrm{nt}$ to $345 \mathrm{nt}$ from $c$-MYC TSS). These demonstrate: (i) DRB treatment (which leads to downregulation of $c-M Y C$ SPEARs) results in the loss of acH2A.Z (middle panel) and TIP60 (right panel) and a partial loss of H2A.Z (left panel); (ii) Restoration of $c-M Y C$ SPEARs (following DRB reversal) leads to reappearance of H2A.Z, acH2A.Z and TIP60; and (iii) Inhibition of TIP60 activity by MG149 and TH1834 prevents the restoration of acH2A.Z, while only slightly affecting = TIP60 and H2A.Z enrichment. Quantitation by qPCR: bars indicate mean \pm s.d. $(n=2)$. 
Ebralidze A.K. et al. text and figure legends

\section{Fig. 5. SPEARs regulate the recycling of the epigenetic acetylation mark on histone H2A.Z.}

a, Outline of experimental design. b, Response of total $c-M Y C$ mRNA and SPEARs to the treatment with TIP60/HAT inhibitor NU 9056. c, Western blot analyses of proteins isolated 4 hours after release from synchrony and treatment with TIP60/HAT inhibitor NU 9056, showing that the global content of H2A.Z is not affected by HAT-inhibitor treatment, in contrast to acH2A.Z, which is highly depleted, i.e. the modification is largely absent. d, Nascent ChIP-qPCRs monitored at the peak of the $c-M Y C$ locus using antibodies to acH2A.Z showing a 7-fold enrichment over total chromatin. e, ChIP-qPCRs for the $c$-MYC locus using antibodies to acH2A.Z following treatment with HAT inhibitor NU 9056; (amplicon located 652 nt to $345 \mathrm{nt}$ upstream of the $c$-MYC TSS). f, Examples of the detected N-terminal peptides from sample "HAT inhibitor (NU 9056)-treated sample (4 hours)". Left Panel: Peptides with all light Lysines and light or heavy acetyl groups (the latter carry the ${ }^{13} \mathrm{C}_{6}$ carbon atoms and add height toward the third isotopic peak in the distribution). When compared to the same N-terminal peptide with no heavy acetyl groups, shown in the RH panel and representing natural abundance of the third isotope in the distribution, the heavy acetyl groups from HAT inhibitor treated sample shows elevated height of the third peak from $37 \%$ of natural distribution (compare to the first ${ }^{12} \mathrm{C}$ peak) to about $67 \%$. Observed change in the third isotope abundance between the two samples shows that in the HAT inhibited sample about $55 \%$ of the N-terminal peptide contain heavy acetyl groups $(37 \% / 67 \%$ x 100\% = 55\%). Right Panel: Mock (DMSO)-treated sample shows the Nterminal peptide (AGGKAGKDSGKAKTK) with three heavy Lysines and the absence of incorporated heavy acetyl groups. The HAT inhibitor (NU9056)-treated sample (4 hrs) shows that the ratio of the $\mathrm{N}$-terminal peptide with three light Lysines (at $\mathrm{m} / \mathrm{z} \sim 510) v s$. the same peptide with all three heavy Lysines (at m/z 524) was measured as 1.8 (see Extended Data Fig. 6c) 
Ebralidze A.K. et al. text and figure legends

Fig. 6. Model: During early S phase, SPEARs interact with both histone H2A.Z and the acetyltransferase TIP60. H2A.Z and TIP60 achieve physical proximity, leading to a high local effective protein concentration ${ }^{60}$ that favors H2A.Z acetylation and exchange with canonical H2A within the nucleosome. In this active chromatin conformation, gene expression is initiated. The incorporated "old" acetylated H2A.Z marks are preserved and transmitted through the generational border.

\section{References}

1. Hanahan, D. \& Weinberg, R.A. Hallmarks of cancer: the next generation. Cell 144, 646-74 (2011).

2. Barski, A. et al. High-resolution profiling of histone methylations in the human genome. Cell 129, 823-37 (2007).

3. Venkatesh, S. \& Workman, J.L. Histone exchange, chromatin structure and the regulation of transcription. Nat Rev Mol Cell Biol 16, 178-89 (2015).

4. Bruce, K. et al. The replacement histone H2A.Z in a hyperacetylated form is a feature of active genes in the chicken. Nucleic Acids Res 33, 5633-9 (2005).

5. Numata, A. et al. Lysine acetyltransferase Tip60 is required for hematopoietic stem cell maintenance. Blood 136, 1735-1747 (2020).

6. Rudnizky, S. et al. H2A.Z controls the stability and mobility of nucleosomes to regulate expression of the LH genes. Nat Commun 7, 12958 (2016).

7. Marques, M., Laflamme, L., Gervais, A.L. \& Gaudreau, L. Reconciling the positive and negative roles of histone H2A.Z in gene transcription. Epigenetics 5, 267-72 (2010).

8. Zovkic, I.B., Paulukaitis, B.S., Day, J.J., Etikala, D.M. \& Sweatt, J.D. Histone H2A.Z subunit exchange controls consolidation of recent and remote memory. Nature 515, 582-6 (2014).

9. Keogh, M.C. et al. The Saccharomyces cerevisiae histone H2A variant Htz1 is acetylated by NuA4. Genes Dev 20, 660-5 (2006).

10. Alabert, C. et al. Two distinct modes for propagation of histone PTMs across the cell cycle. Genes Dev 29, 585-90 (2015).

11. Zenk, F. et al. Germ line-inherited H3K27me3 restricts enhancer function during maternal-tozygotic transition. Science 357, 212-216 (2017).

12. Reveron-Gomez, N. et al. Accurate Recycling of Parental Histones Reproduces the Histone Modification Landscape during DNA Replication. Mol Cell 72, 239-249 e5 (2018).

13. Rinn, J.L. et al. Functional demarcation of active and silent chromatin domains in human HOX loci by noncoding RNAs. Cell 129, 1311-23 (2007).

14. Yelin, R. et al. Widespread occurrence of antisense transcription in the human genome. Nat Biotechnol 21, 379-86 (2003).

15. Costa, F.F. Non-coding RNAs, epigenetics and complexity. Gene (2008). 
Ebralidze A.K. et al. text and figure legends

16. Guttman, M. et al. Chromatin signature reveals over a thousand highly conserved large noncoding RNAs in mammals. Nature 458, 223-7 (2009).

17. Khalil, A.M. et al. Many human large intergenic noncoding RNAs associate with chromatinmodifying complexes and affect gene expression. Proc Natl Acad Sci U S A 106, 11667-72 (2009).

18. Ponting, C.P., Oliver, P.L. \& Reik, W. Evolution and functions of long noncoding RNAs. Cell 136, 629-41 (2009).

19. Mercer, T.R., Dinger, M.E. \& Mattick, J.S. Long non-coding RNAs: insights into functions. Nat Rev Genet 10, 155-9 (2009).

20. Wilusz, J.E., Sunwoo, H. \& Spector, D.L. Long noncoding RNAs: functional surprises from the RNA world. Genes Dev 23, 1494-504 (2009).

21. Jandura, A. \& Krause, H.M. The New RNA World: Growing Evidence for Long Noncoding RNA Functionality. Trends Genet 33, 665-676 (2017).

22. Huarte, M. The emerging role of lncRNAs in cancer. Nat Med 21, 1253-61 (2015).

23. Huarte, M. et al. A large intergenic noncoding RNA induced by p53 mediates global gene repression in the p53 response. Cell 142, 409-19 (2010).

24. Ebralidze, A., Wang, Y., Petkova, V., Ebralidse, K. \& Junghans, R.P. RNA leaching of transcription factors disrupts transcription in myotonic dystrophy. Science 303, 383-7 (2004).

25. Ebralidze, A.K. et al. PU.1 expression is modulated by the balance of functional sense and antisense RNAs regulated by a shared cis-regulatory element. Genes Dev 22, 2085-92 (2008).

26. Carrieri, C. et al. Long non-coding antisense RNA controls Uchl1 translation through an embedded SINEB2 repeat. Nature 491, 454-7 (2012).

27. Gupta, R.A. et al. Long non-coding RNA HOTAIR reprograms chromatin state to promote cancer metastasis. Nature 464, 1071-6 (2010).

28. Zucchelli, S. et al. SINEUPs: A new class of natural and synthetic antisense long non-coding RNAs that activate translation. RNA Biol 12, 771-9 (2015).

29. Hung, T. et al. Extensive and coordinated transcription of noncoding RNAs within cell-cycle promoters. Nat Genet 43, 621-9 (2011).

30. Bose, D.A. et al. RNA Binding to CBP Stimulates Histone Acetylation and Transcription. Cell 168, 135-149 e22 (2017).

31. Rodriguez-Campos, A. \& Azorin, F. RNA is an integral component of chromatin that contributes to its structural organization. PLoS One 2, e1182 (2007).

32. Werner, M.S. \& Ruthenburg, A.J. Nuclear Fractionation Reveals Thousands of ChromatinTethered Noncoding RNAs Adjacent to Active Genes. Cell Rep 12, 1089-98 (2015).

33. Tsai, M.C., Spitale, R.C. \& Chang, H.Y. Long intergenic noncoding RNAs: new links in cancer progression. Cancer Res 71, 3-7 (2011).

34. Martianov, I., Ramadass, A., Serra Barros, A., Chow, N. \& Akoulitchev, A. Repression of the human dihydrofolate reductase gene by a non-coding interfering transcript. Nature 445, 66670 (2007).

35. Tripathi, V. et al. The nuclear-retained noncoding RNA MALAT1 regulates alternative splicing by modulating SR splicing factor phosphorylation. Mol Cell 39, 925-38 (2010).

36. Lande-Diner, L., Zhang, J. \& Cedar, H. Shifts in replication timing actively affect histone acetylation during nucleosome reassembly. Mol Cell 34, 767-74 (2009).

37. Salic, A. \& Mitchison, T.J. A chemical method for fast and sensitive detection of DNA synthesis in vivo. Proc Natl Acad Sci U S A 105, 2415-20 (2008).

38. Buck, S.B. et al. Detection of S-phase cell cycle progression using 5-ethynyl-2'-deoxyuridine incorporation with click chemistry, an alternative to using 5-bromo-2'-deoxyuridine antibodies. Biotechniques 44, 927-9 (2008). 
Ebralidze A.K. et al. text and figure legends

39. Kress, T.R., Sabo, A. \& Amati, B. MYC: connecting selective transcriptional control to global RNA production. Nat Rev Cancer 15, 593-607 (2015).

40. Vangala, R.K. et al. The myeloid master regulator transcription factor PU.1 is inactivated by AML1-ETO in t(8;21) myeloid leukemia. Blood 101, 270-7 (2003).

41. Pelicci, P.G., Lanfrancone, L., Brathwaite, M.D., Wolman, S.R. \& Dalla-Favera, R. Amplification of the c-myb oncogene in a case of human acute myelogenous leukemia. Science 224, 1117-21 (1984).

42. Millar, C.B., Xu, F., Zhang, K. \& Grunstein, M. Acetylation of H2AZ Lys 14 is associated with genome-wide gene activity in yeast. Genes Dev 20, 711-22 (2006).

43. Hofacker, I.L. Vienna RNA secondary structure server. Nucleic Acids Res 31, 3429-31 (2003).

44. Hofacker, I.L., Bernhart, S.H. \& Stadler, P.F. Alignment of RNA base pairing probability matrices. Bioinformatics 20, 2222-7 (2004).

45. Di Ruscio, A. et al. DNMT1-interacting RNAs block gene-specific DNA methylation. Nature 503, 371-6 (2013).

46. Guttman, M. et al. lincRNAs act in the circuitry controlling pluripotency and differentiation. Nature 477, 295-300 (2011).

47. Chen, P.B., Chen, H.V., Acharya, D., Rando, O.J. \& Fazzio, T.G. R loops regulate promoterproximal chromatin architecture and cellular differentiation. Nat Struct Mol Biol 22, 9991007 (2015).

48. Zhao, J., Sun, B.K., Erwin, J.A., Song, J.J. \& Lee, J.T. Polycomb proteins targeted by a short repeat RNA to the mouse X chromosome. Science 322, 750-6 (2008).

49. Ljungman, M., Zhang, F., Chen, F., Rainbow, A.J. \& McKay, B.C. Inhibition of RNA polymerase II as a trigger for the p53 response. Oncogene 18, 583-92 (1999).

50. Sirbu, B.M. et al. Analysis of protein dynamics at active, stalled, and collapsed replication forks. Genes Dev 25, 1320-7 (2011).

51. Xu, C. \& Corces, V.G. Genome-Wide Mapping of Protein-DNA Interactions on Nascent Chromatin. Methods Mol Biol 1766, 231-238 (2018).

52. $\mathrm{Xu}, \mathrm{C} . \&$ Corces, V.G. Nascent DNA methylome mapping reveals inheritance of hemimethylation at CTCF/cohesin sites. Science 359, 1166-1170 (2018).

53. Gao, C. et al. Rational design and validation of a Tip60 histone acetyltransferase inhibitor. Sci Rep 4, 5372 (2014).

54. Ghizzoni, M. et al. 6-alkylsalicylates are selective Tip60 inhibitors and target the acetyl-CoA binding site. Eur J Med Chem 47, 337-44 (2012).

55. Mukherjee, R. \& Molloy, G.R. 5,6-Dichloro-1-beta-D-ribofuranosylbenzimidazole inhibits transcription of the beta-hemoglobin gene in vivo at initiation. J Biol Chem 262, 13697-705 (1987).

56. Listerman, I., Bledau, A.S., Grishina, I. \& Neugebauer, K.M. Extragenic accumulation of RNA polymerase II enhances transcription by RNA polymerase III. PLoS Genet 3, e212 (2007).

57. Ahmad, K. \& Henikoff, S. The histone variant H3.3 marks active chromatin by replicationindependent nucleosome assembly. Mol Cell 9, 1191-200 (2002).

58. Lu, Q. et al. Epigenetics, disease, and therapeutic interventions. Ageing Res Rev 5, 449-67 (2006).

59. Qi, Y. et al. HEDD: the human epigenetic drug database. Database (Oxford) 2016(2016).

60. Rippe, K., von Hippel, P.H. \& Langowski, J. Action at a distance: DNA-looping and initiation of transcription. Trends Biochem Sci 20, 500-6 (1995). 
Ebralidze A.K. et al. text and figure legends

Acknowledgements: This work was supported by the award of NCI R35 CA197697, R01DK103858, W81XWH-15-1-0161, and P01HL131477-01A1 to DGT; and R50 CA211304 to AKE; ADR was supported by NCI R00 CA188595, W81XWH-20-1-0518, the Italian Association for Cancer Research (AIRC) and the Giovanni Armenise-Harvard Foundation; AW was supported by the Belgian American Educational Foundation (BAEF) and by Wallonie-Bruxelles International (WBI); K01CA222707 was awarded to BQT; TB was supported by the National Research Foundation and the Singapore Ministry of Education under its Centres of Excellence initiative. This work was also supported by the Singapore Ministry of Health's National Medical Research Council under its Singapore Translational Research (STaR) Investigator Award; by the National Research Foundation Singapore and the Singapore Ministry of Education under its Research Centres of Excellence initiative; and by the Singapore Ministry of Education Academic Research Fund Tier 3, grant number MOE2014-T3-1-006. We thank Bruno Amati for thorough discussion and insightful comments on the manuscript and for providing the TIP60 antibodies; likewise Pier Paolo Pandolfi for critical reading of the manuscript and all the members of the Tenen Laboratory for the helpful suggestions.

Author Contributions: AKE, ADR, CCR and DGT conceived and designed the study and wrote the manuscript; AKE, SU, HZ, ADR, LY, DT, SU, EM, AW, BQT, RC, MRS, HZ, YZ performed experiments; MAB performed bioinformatics and statistical analyses, and analyzed RNA-Seq and RIP-Seq data; VEA, RTM, MAB and TB analyzed ChIP-Seq data, EM and VP performed the RNA motif discovery, BB and DK analyzed Mass Spectrometry datasets. 


\section{Identification of SPEARS} (Nuclear RNA-Seq) (a) Nuclear RNAs

a

Alignment Early S-phase nascent promoter RNAs (nasRNA-Seq)

Early S-phase nascent promoter RNAs correlate with high expressed genes

$(\mathbf{a}, \mathbf{b})$

$(c-e)$

(c-e)

(f)

$(g, h)$

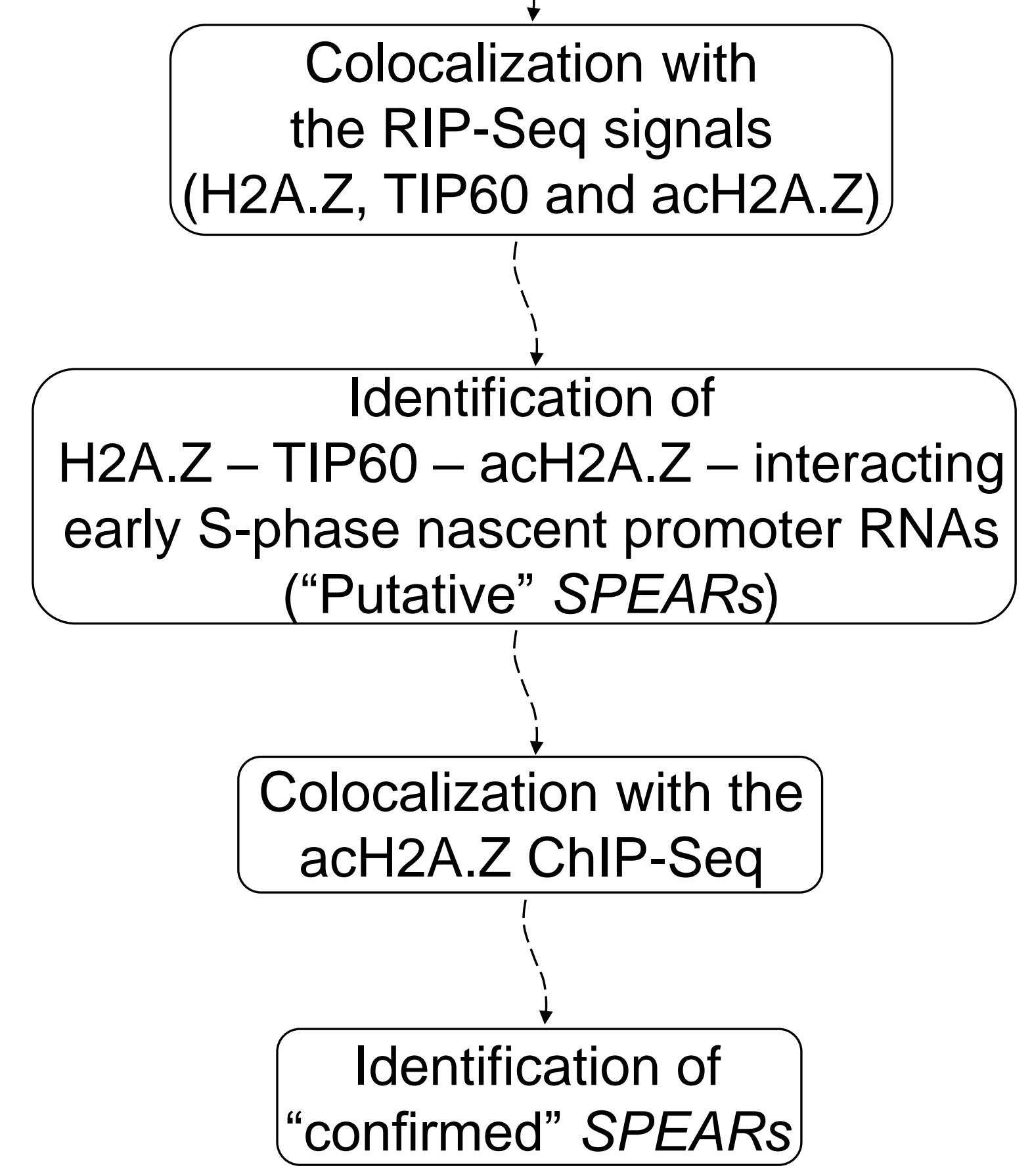

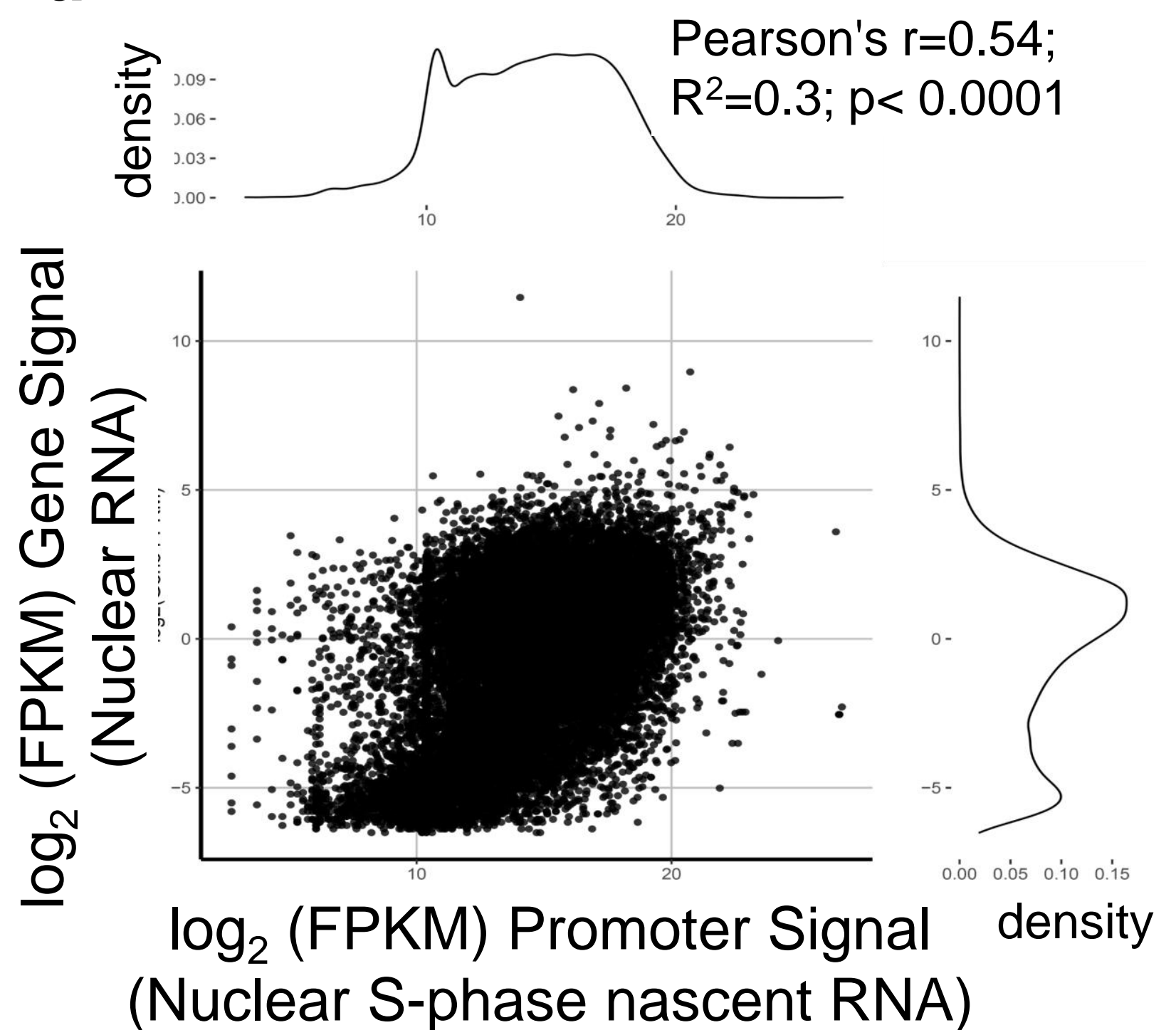
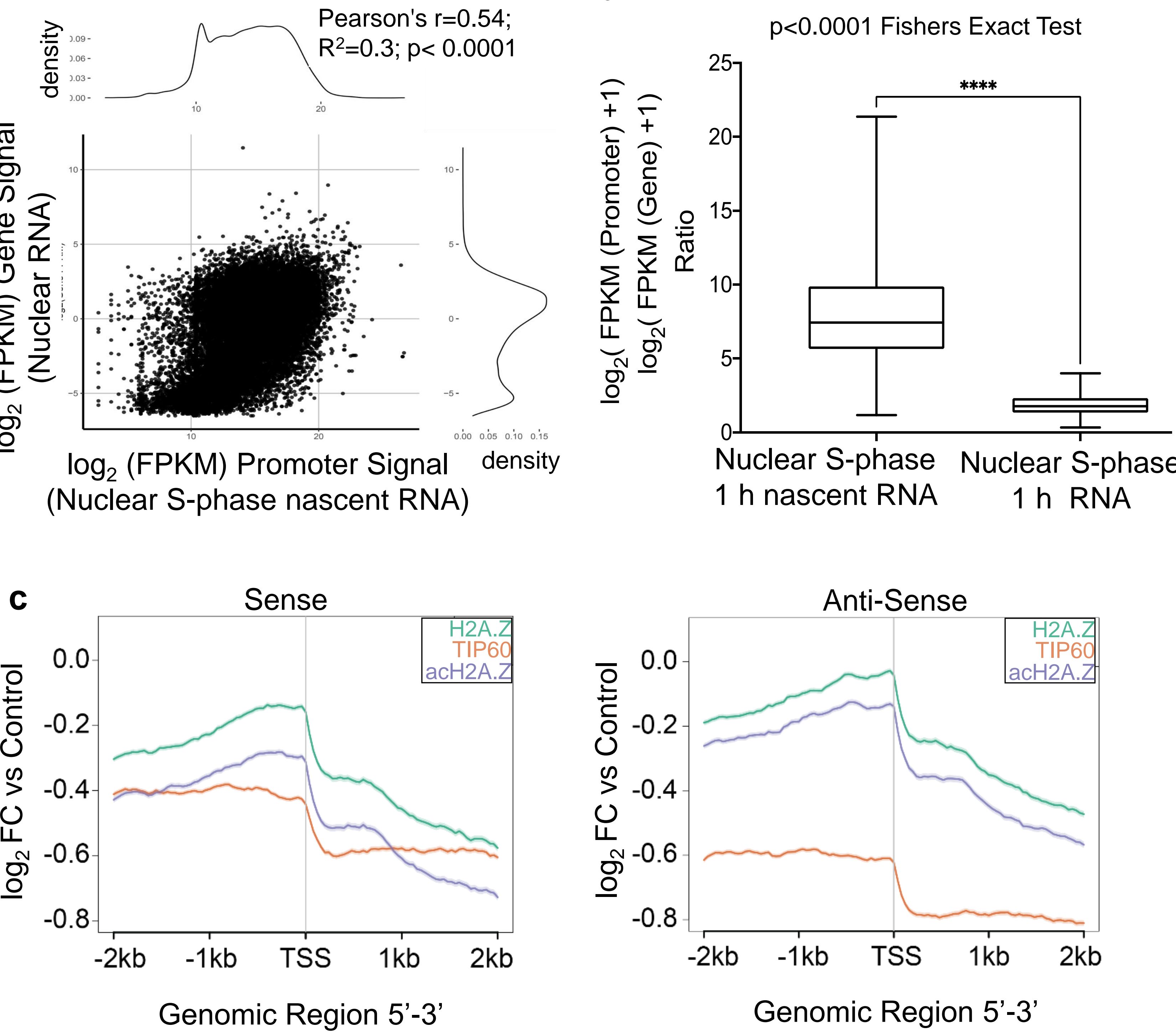

d

H2A.Z

TIP60

$33,44710,440 \quad 114,750$

40,326

$53,666 \quad 19,370$

56,788

acH2A.Z

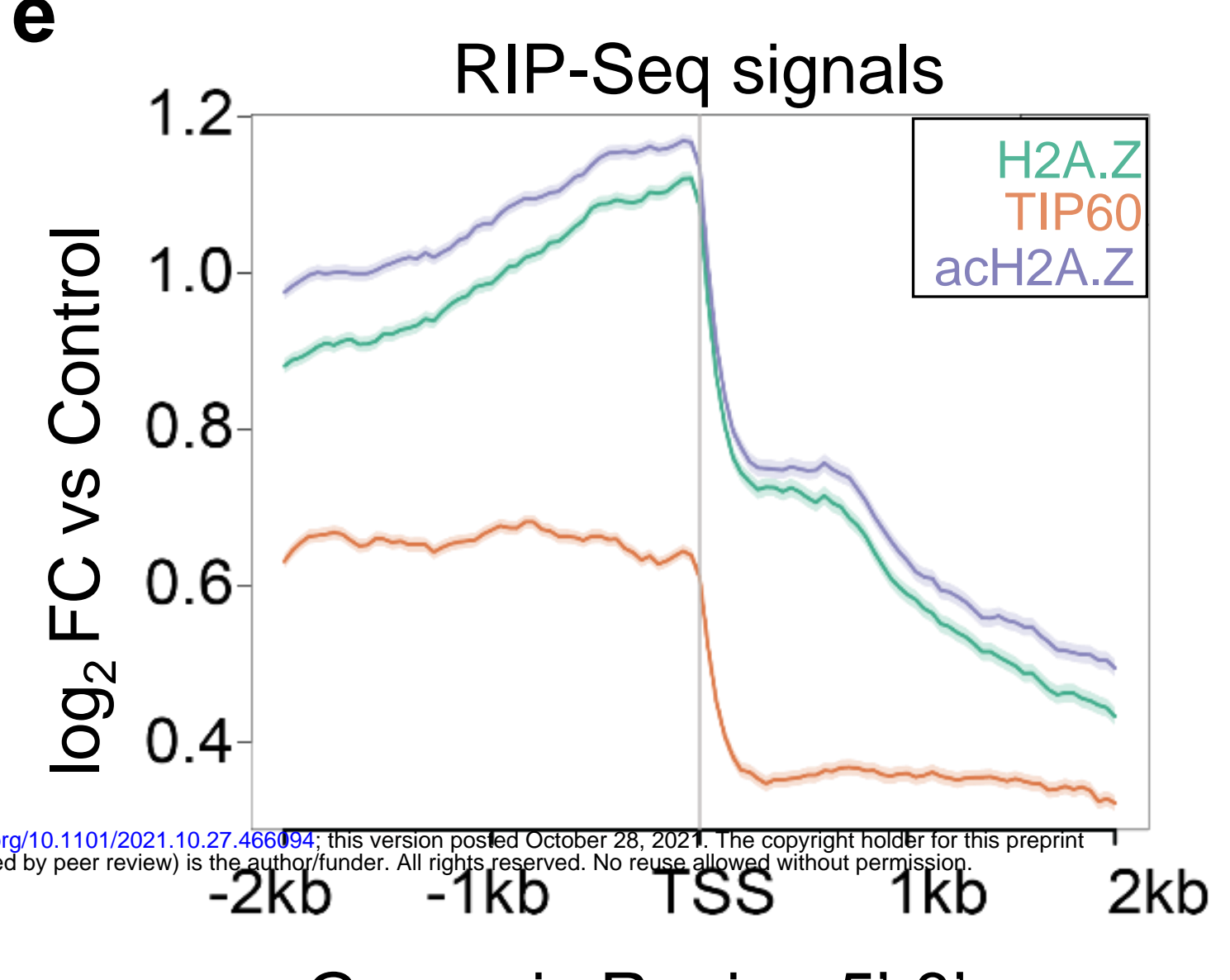

Genomic Region 5'-3'
Select peaks within

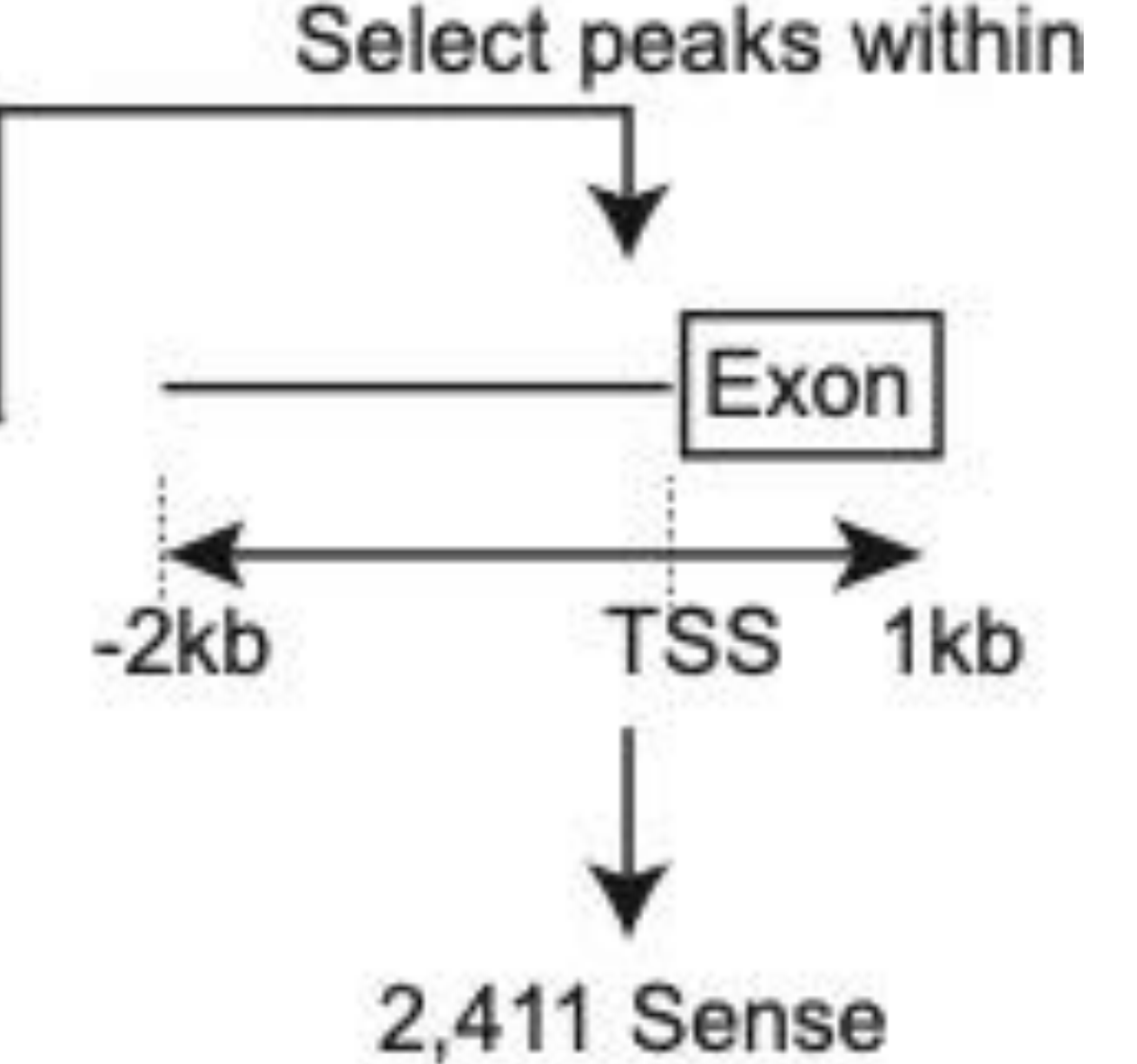

f

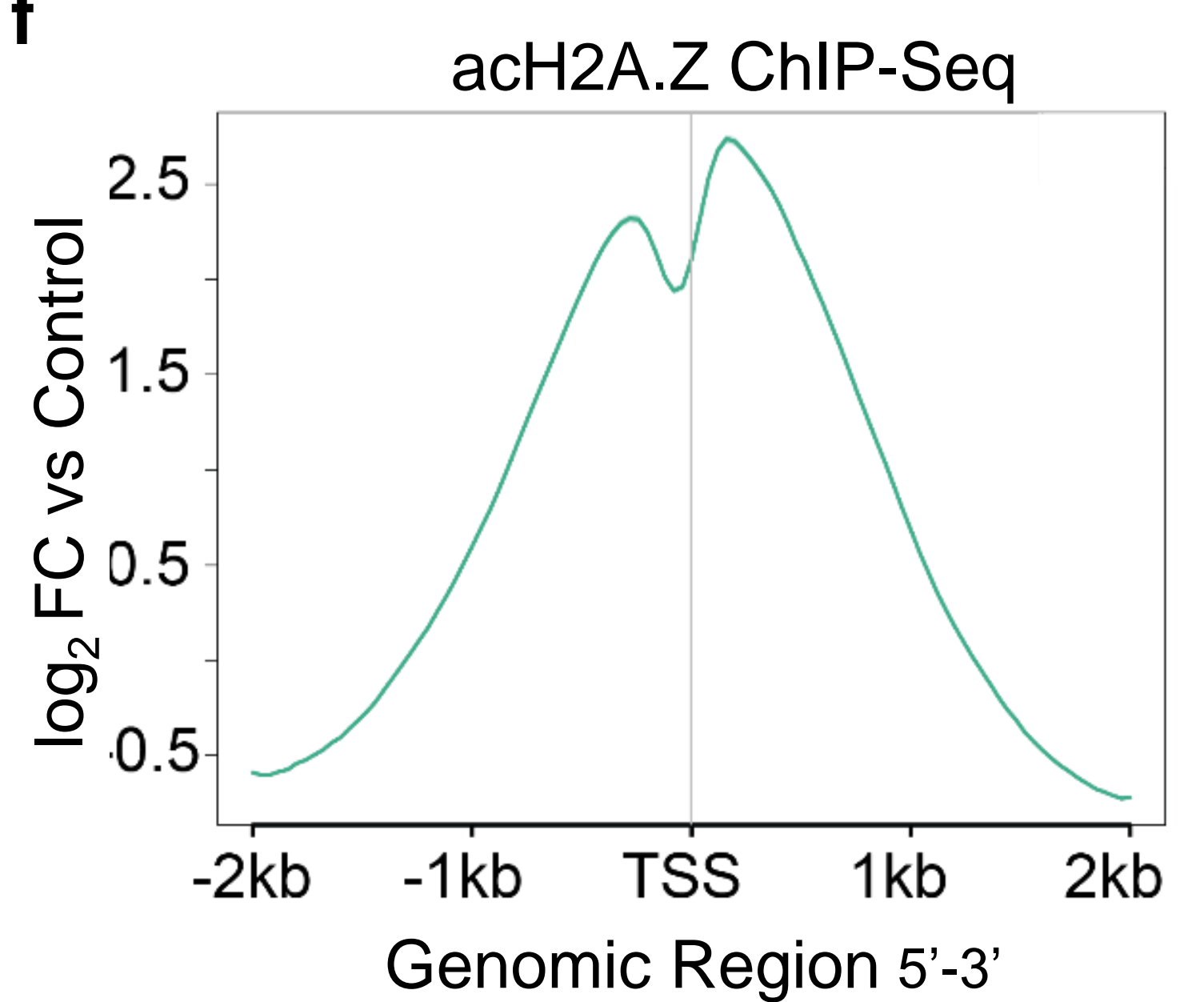

H2A.Z

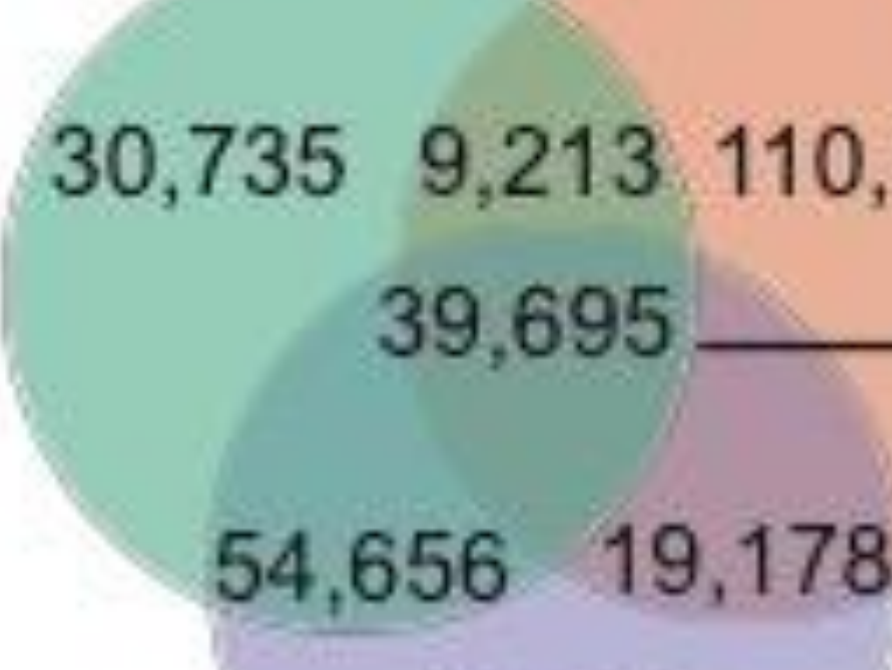

58,255

acH2A.Z
TIP60

Select peaks within

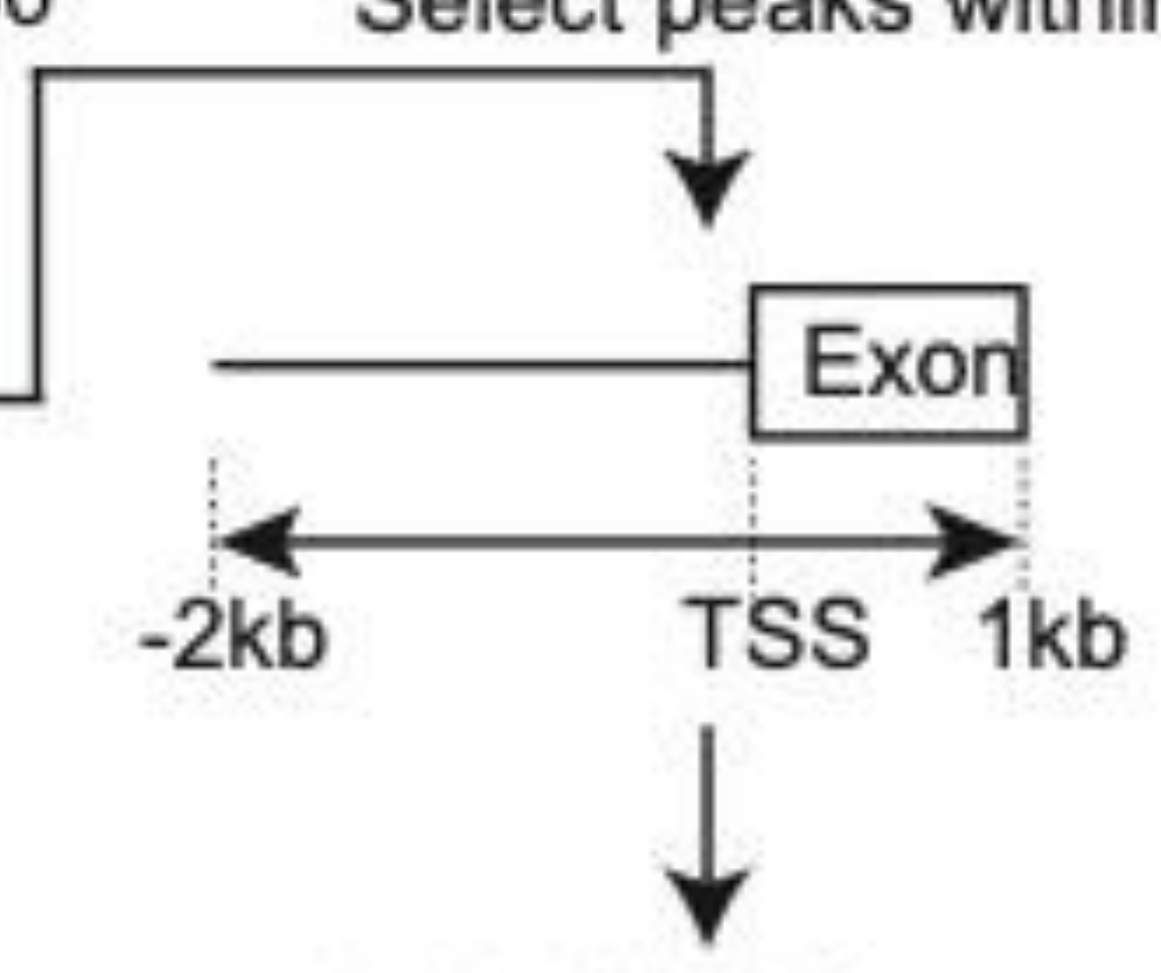

2,278 Anti-Sense

h

RIP-Seq signals

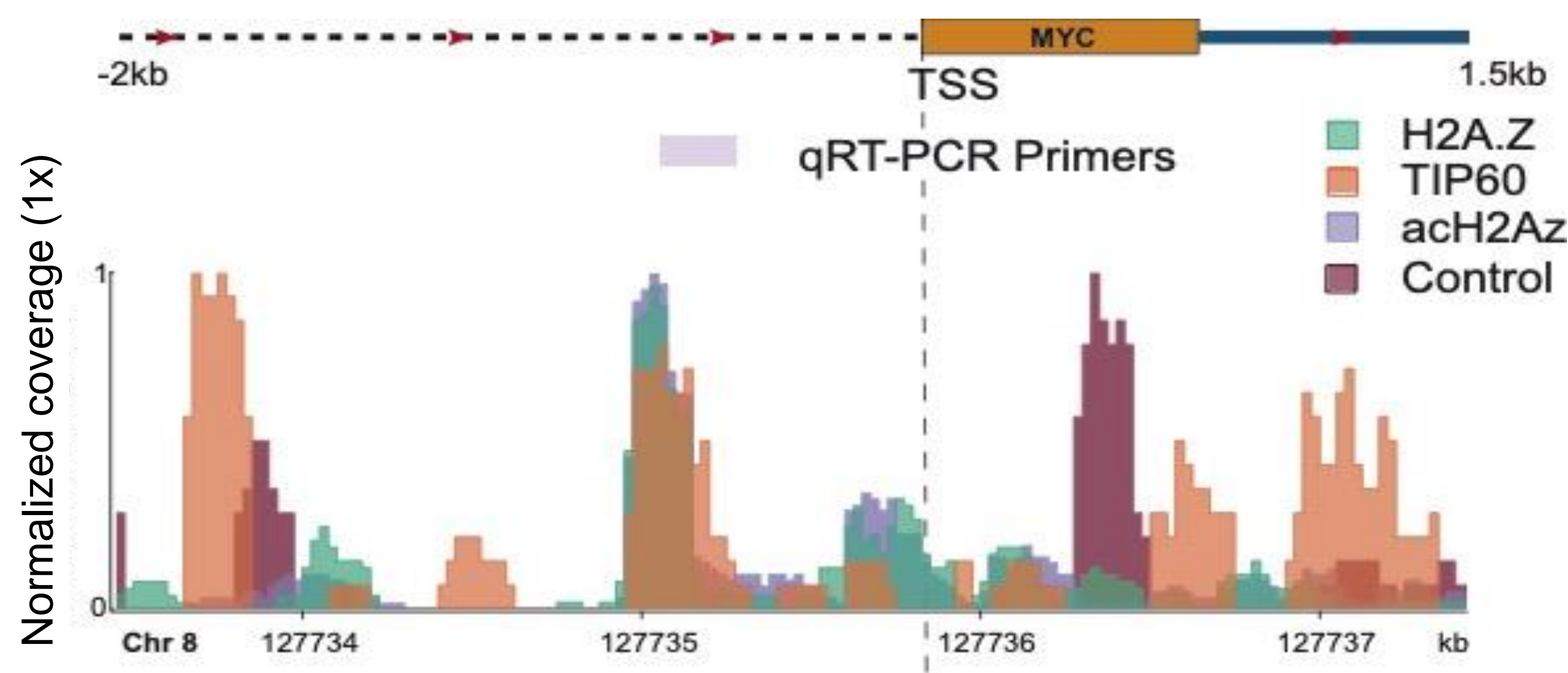

ChIP-Seq signals

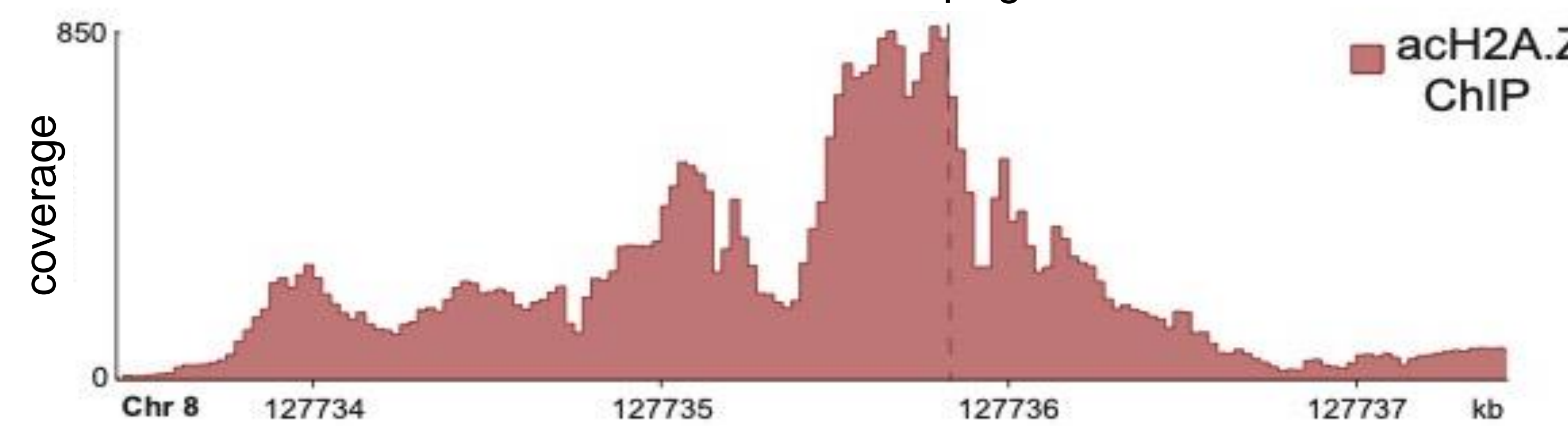

g ChIP-Seq signals

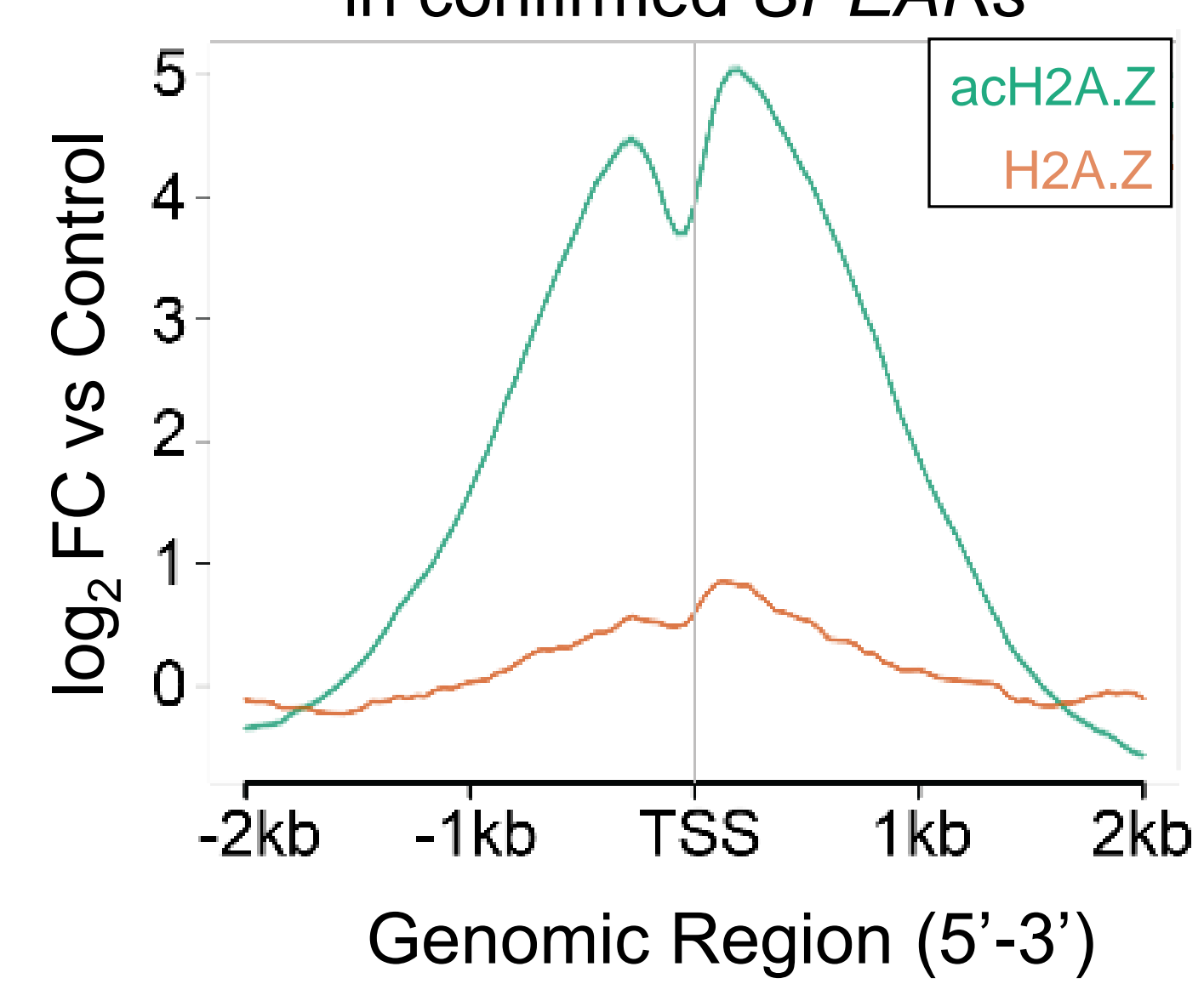




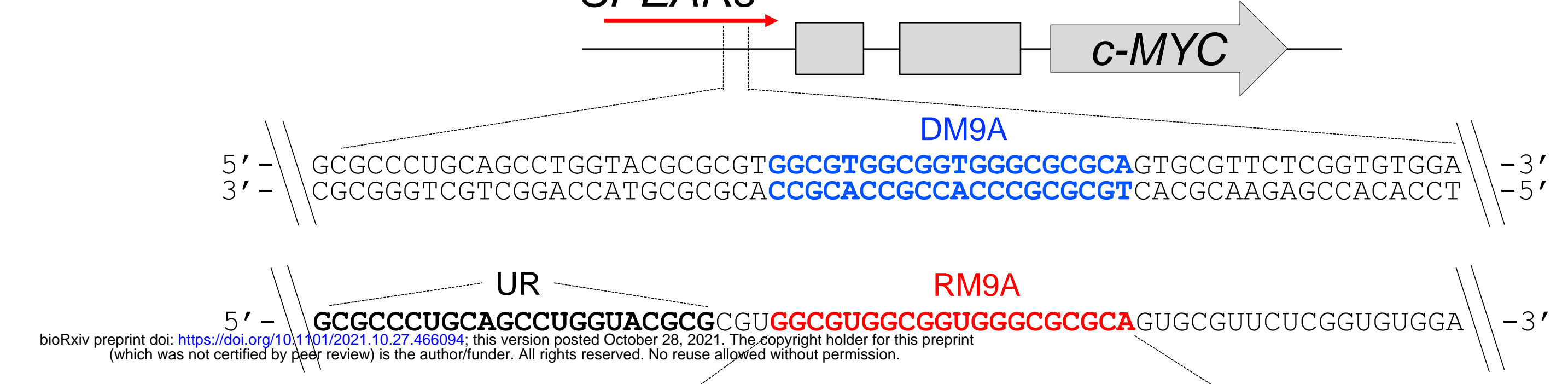

\section{GGUGUEGUGGCUCACCCUG}

b

e

RM9A Mut RM9A

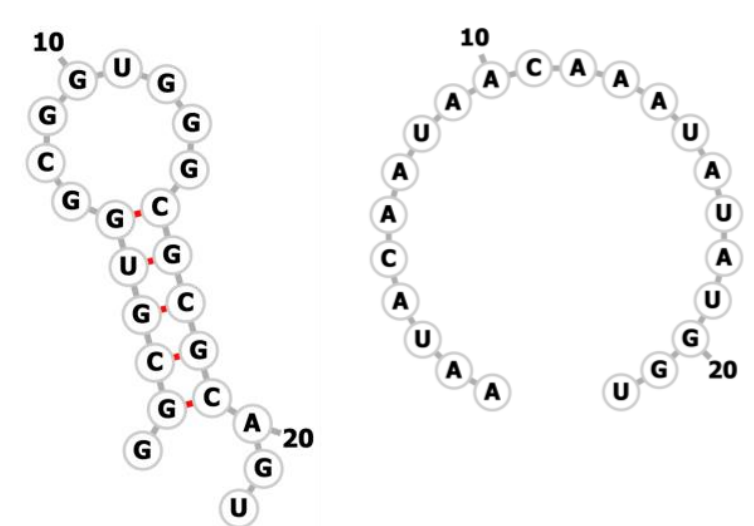

Distance from the center (summit)

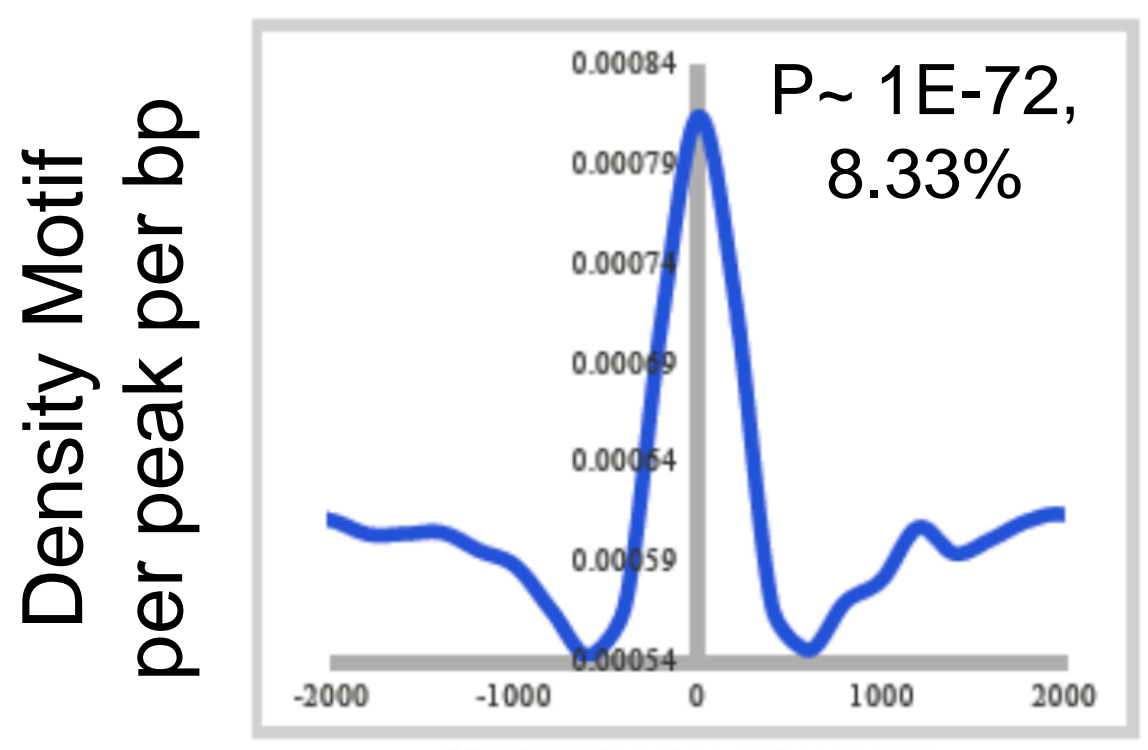

C

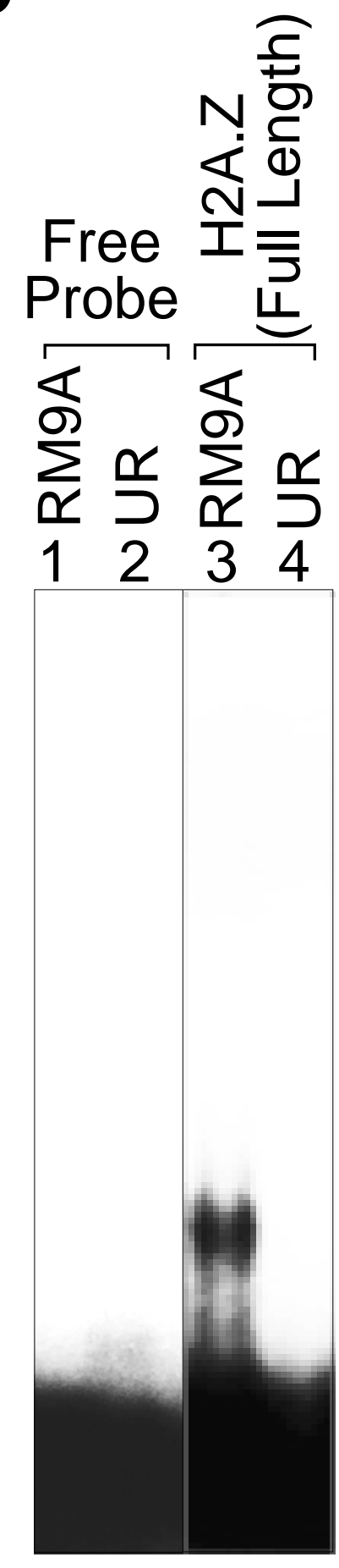

Free

H2A.Z acH2A.Z \& peptide peptide ㄴ $\Sigma \sum_{\Sigma} \sum$ 일 5678910111213

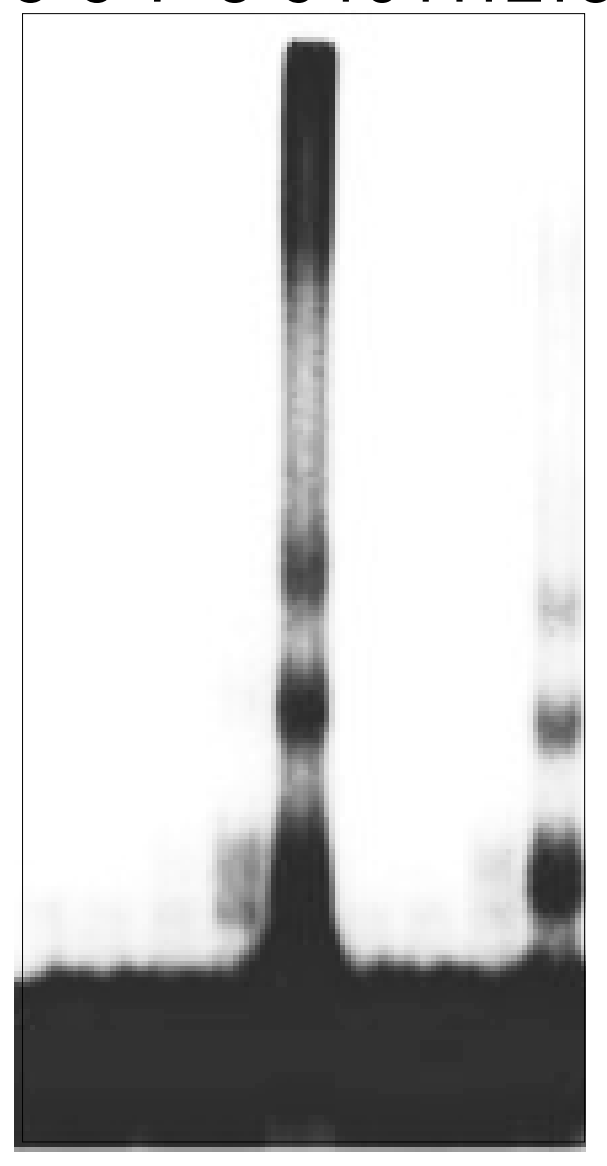

d

(n)

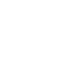




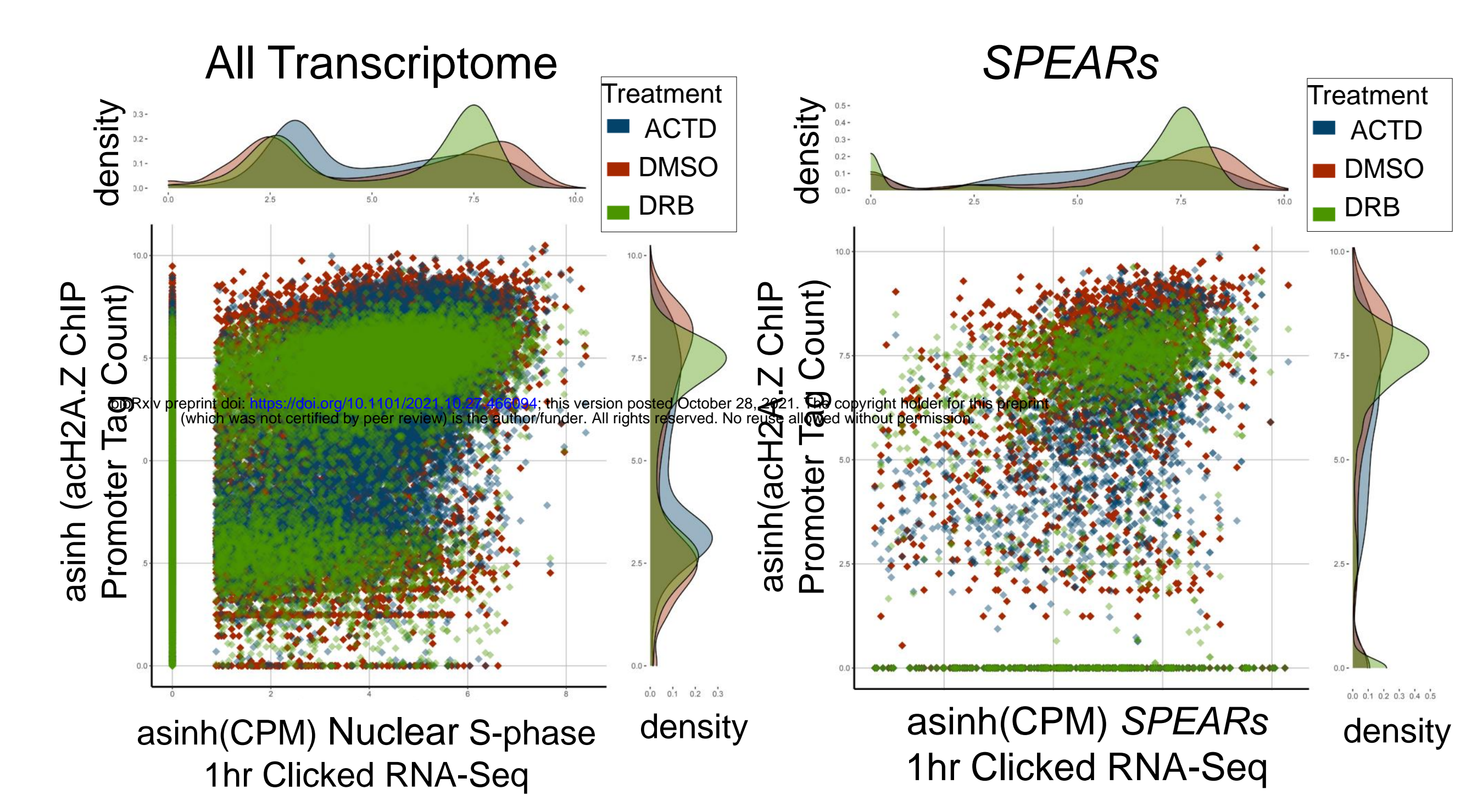

\section{b}
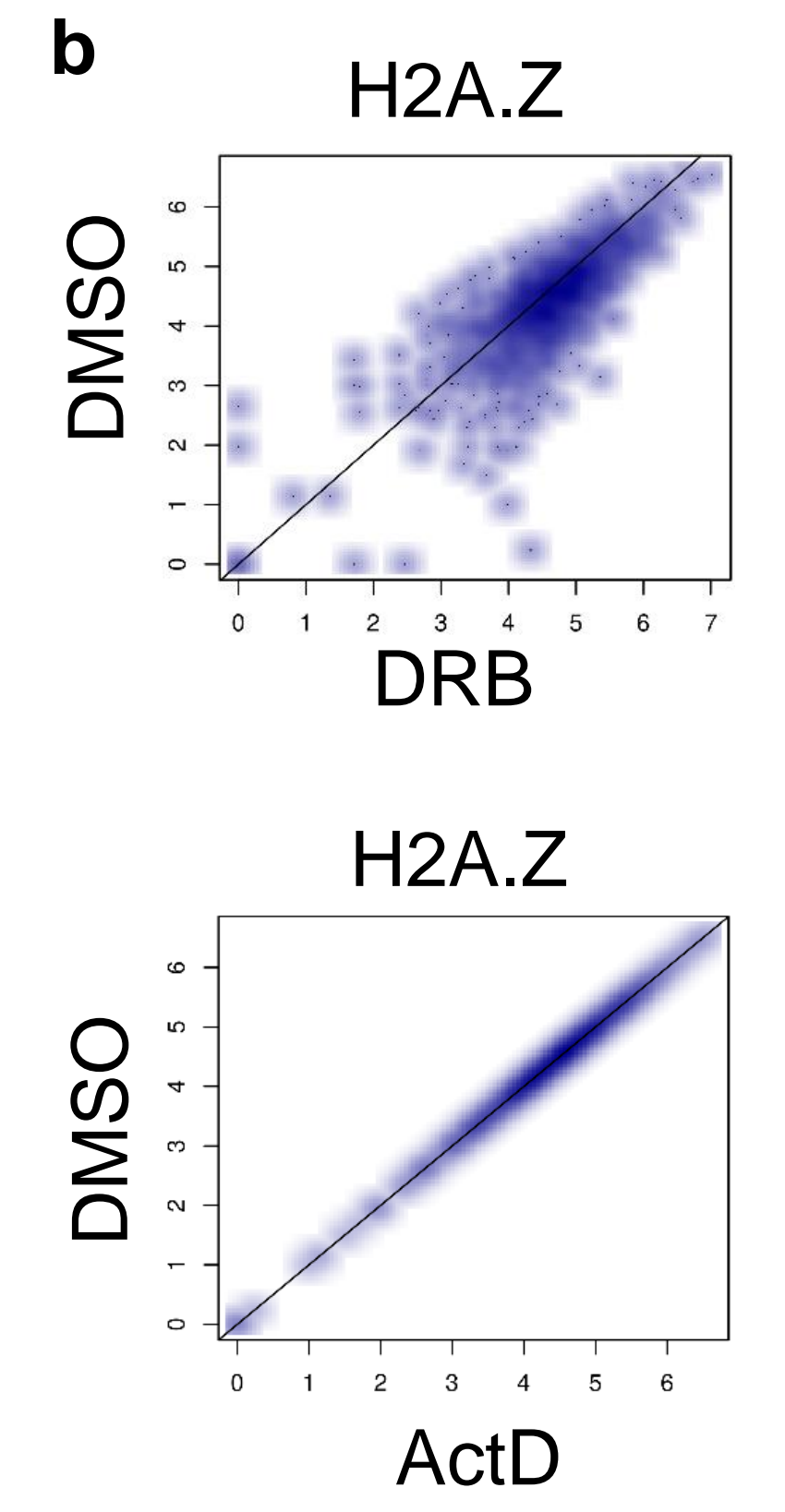

acH2A.Z

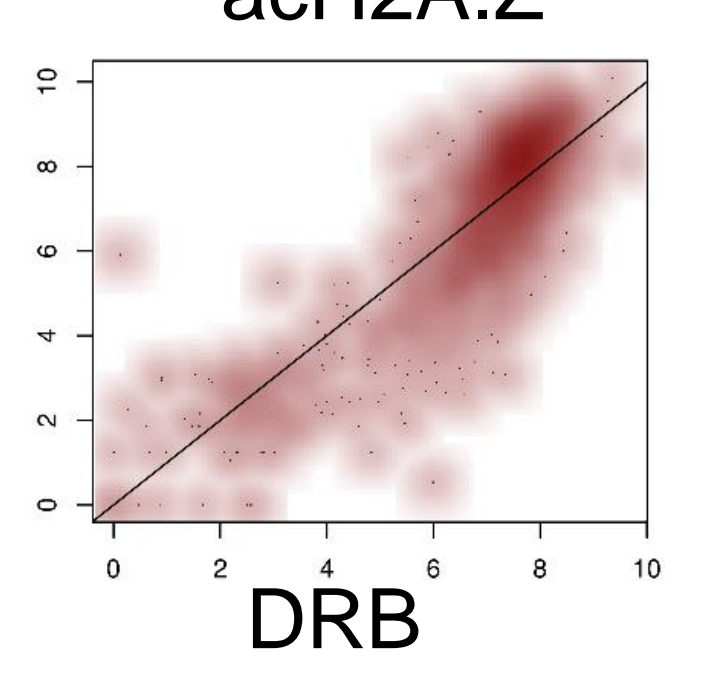

acH2A.Z

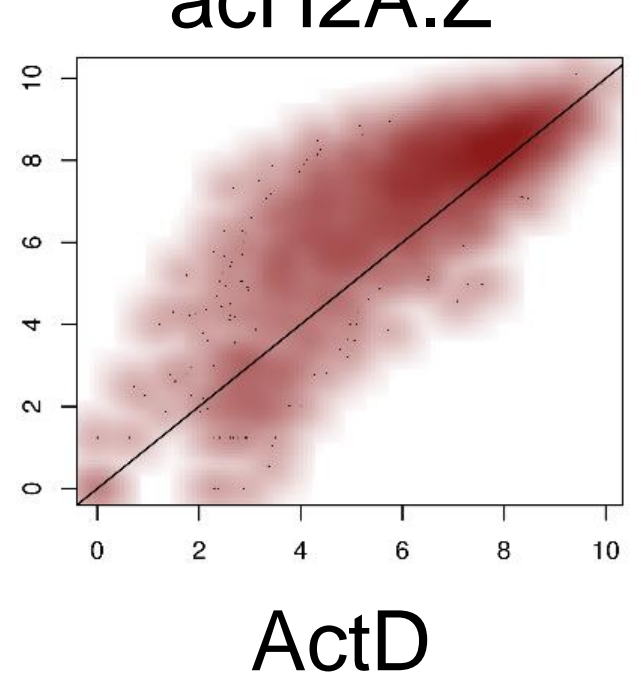

acH2AZ ChIP-Seq

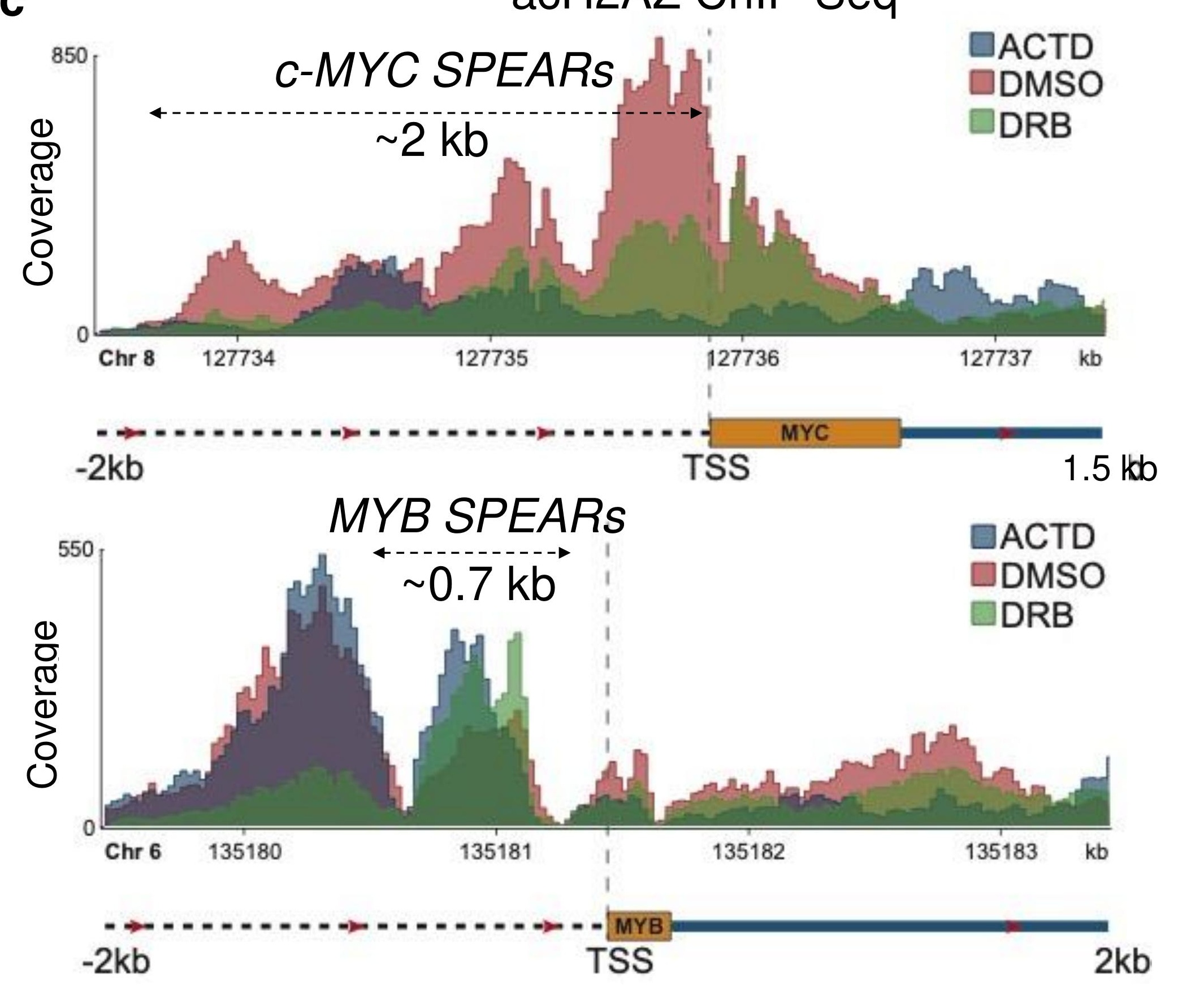

e

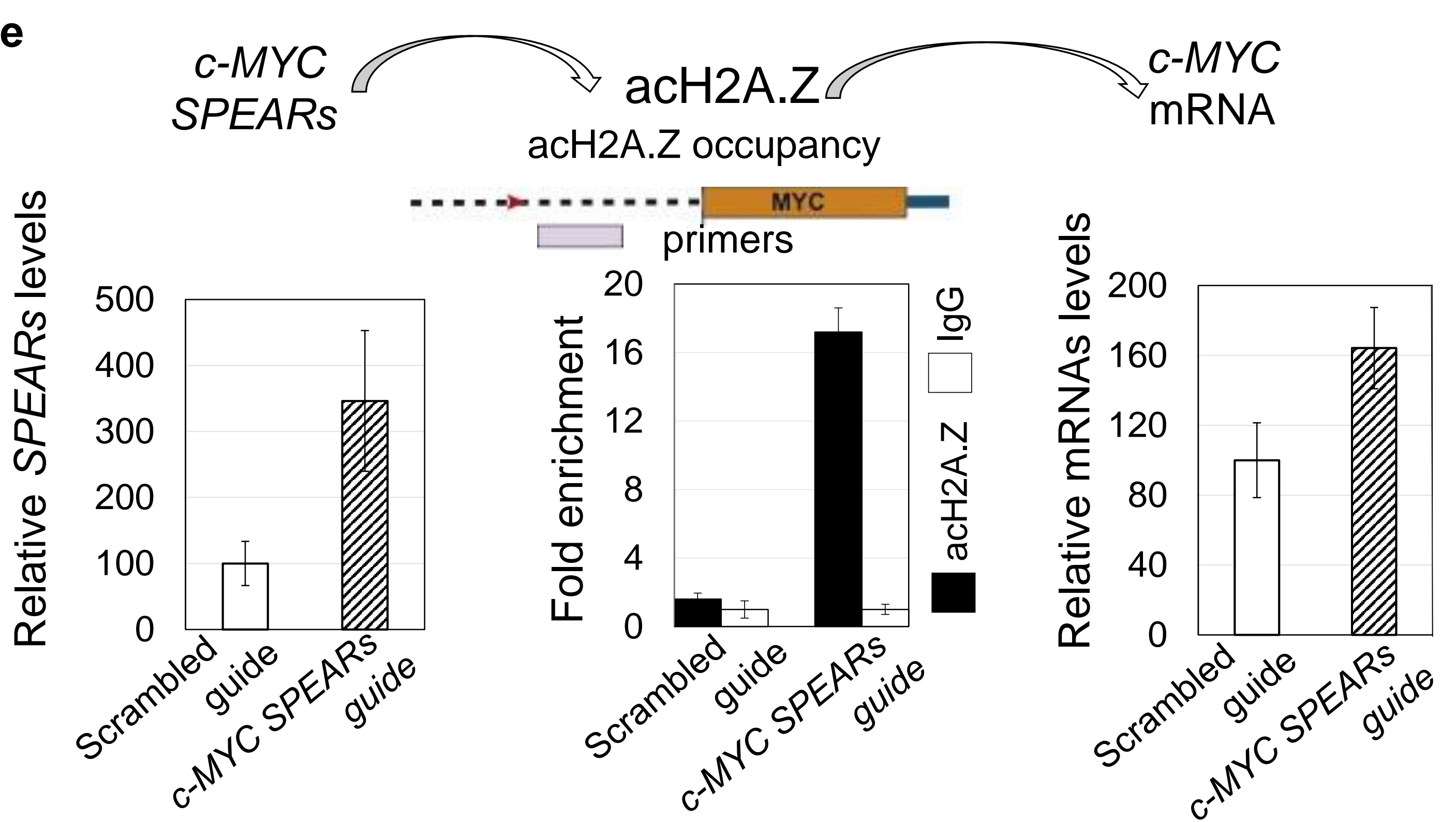

\section{d}
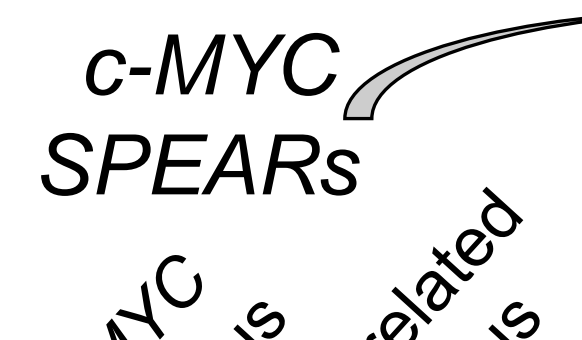

$\frac{\infty}{d}$

$\frac{1}{9} 14$

崩 100

के

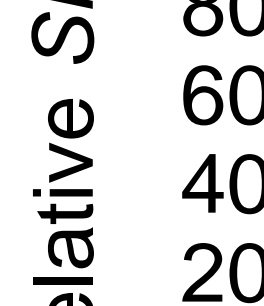

$\begin{array}{lr}\frac{\pi}{0} & 20 \\ & 0\end{array}$

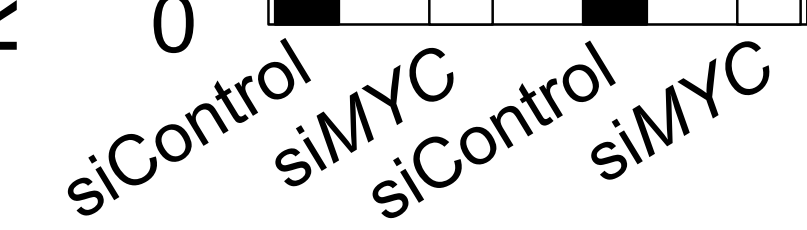

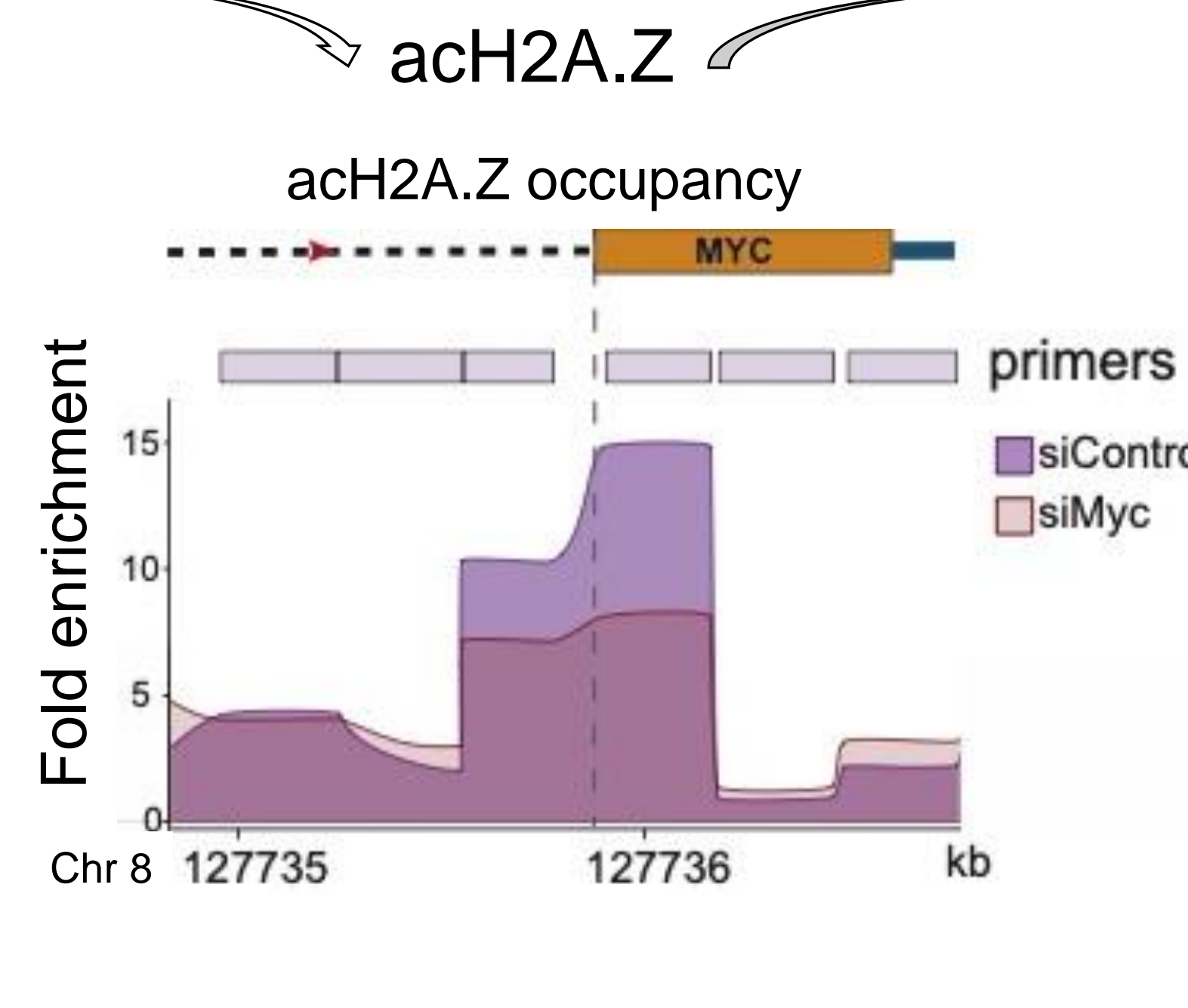

\section{$c-M Y C$}

mRNA

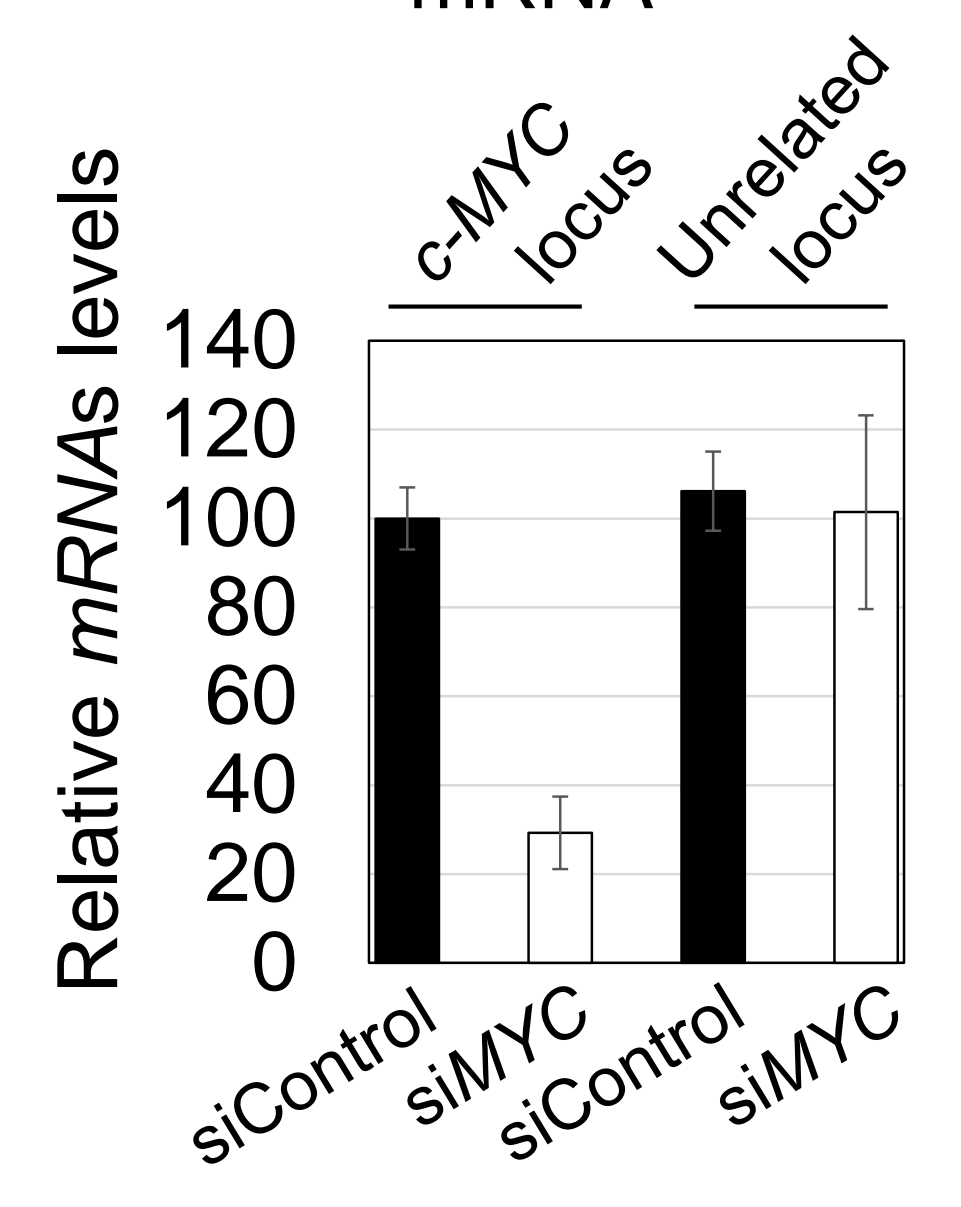


a Culture the cells in Start synchronization Supplement the SILAC SILAC media; of the cells in the SILAC media corporation of "heavy"

${ }^{15} \mathrm{~N}_{2}{ }^{13} \mathrm{C}_{2}$-Lysines (start after day 4) (day 1)

\section{क्व}

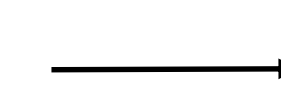

media with ${ }^{13} \mathrm{C}_{6}$-glucose acetyl-coA

\section{उ3} to produce heavy labeled

(start after day 5; for $24 \mathrm{~h}$ )

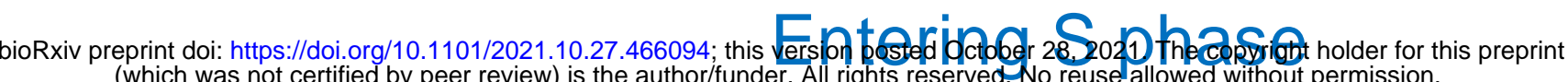

Release into S phase; grow cells in normal medium supplemented with: 1) DMSO; 4 hrs; and

2) HATs Inhibitor NU 9056; 4 hrs

\section{DMSO (mock) treatment}

HATs inhibitor NU 9056 treatment $\Leftrightarrow$ d

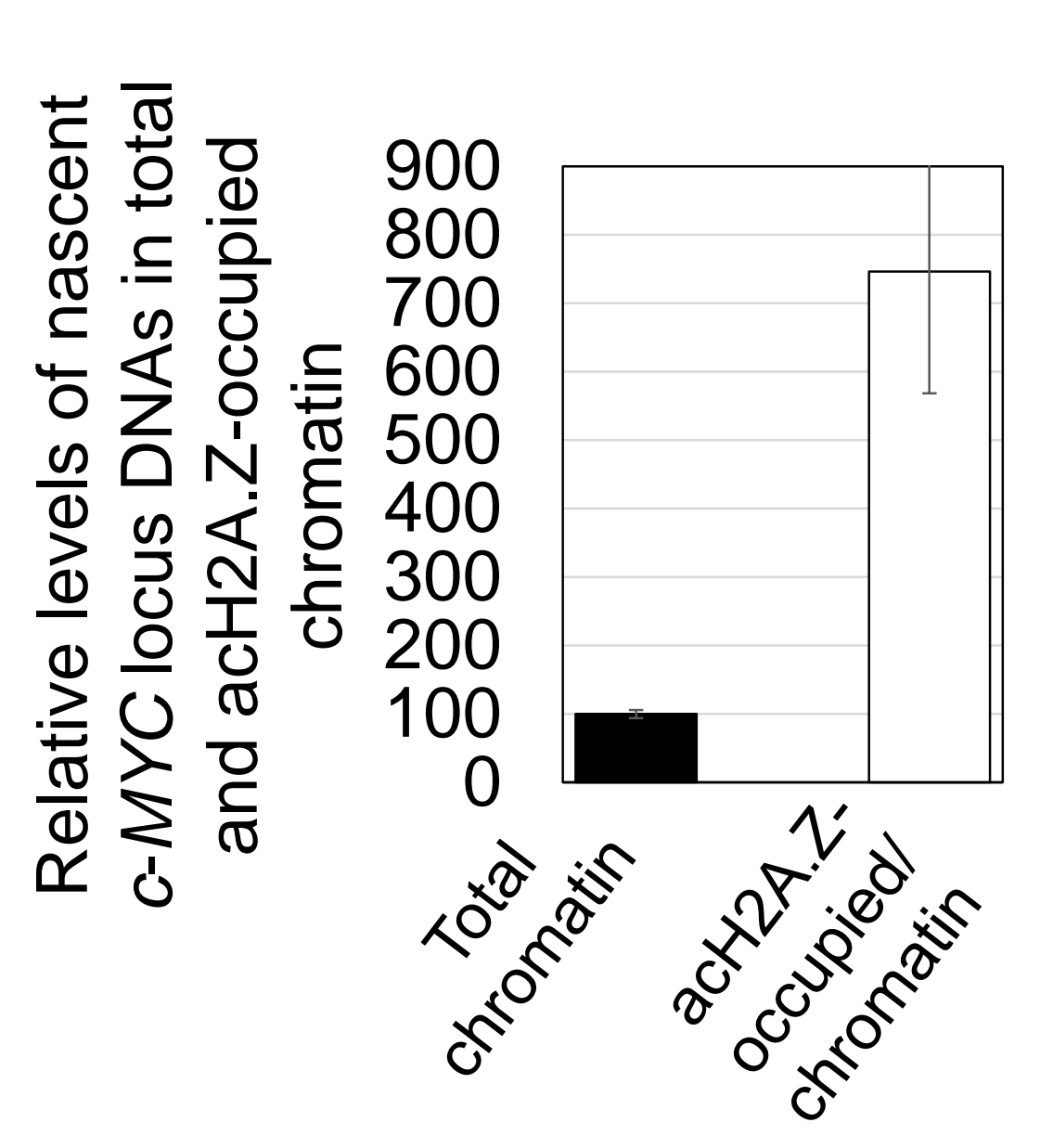

Crosslink the cells and perform ChIP with the antibodies to acetylated H2A.Z. Collect immonoprecipitates and analyze by ChIP-PCR and mass spectrometry

e

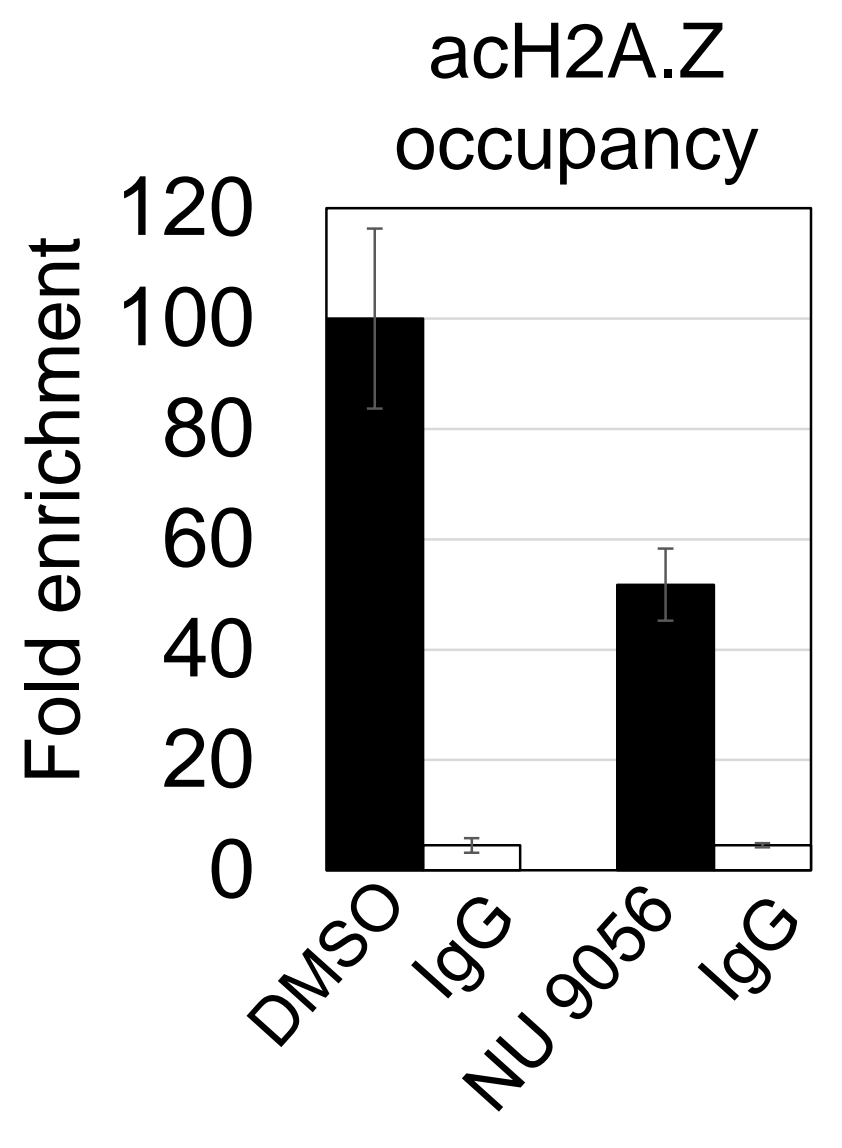

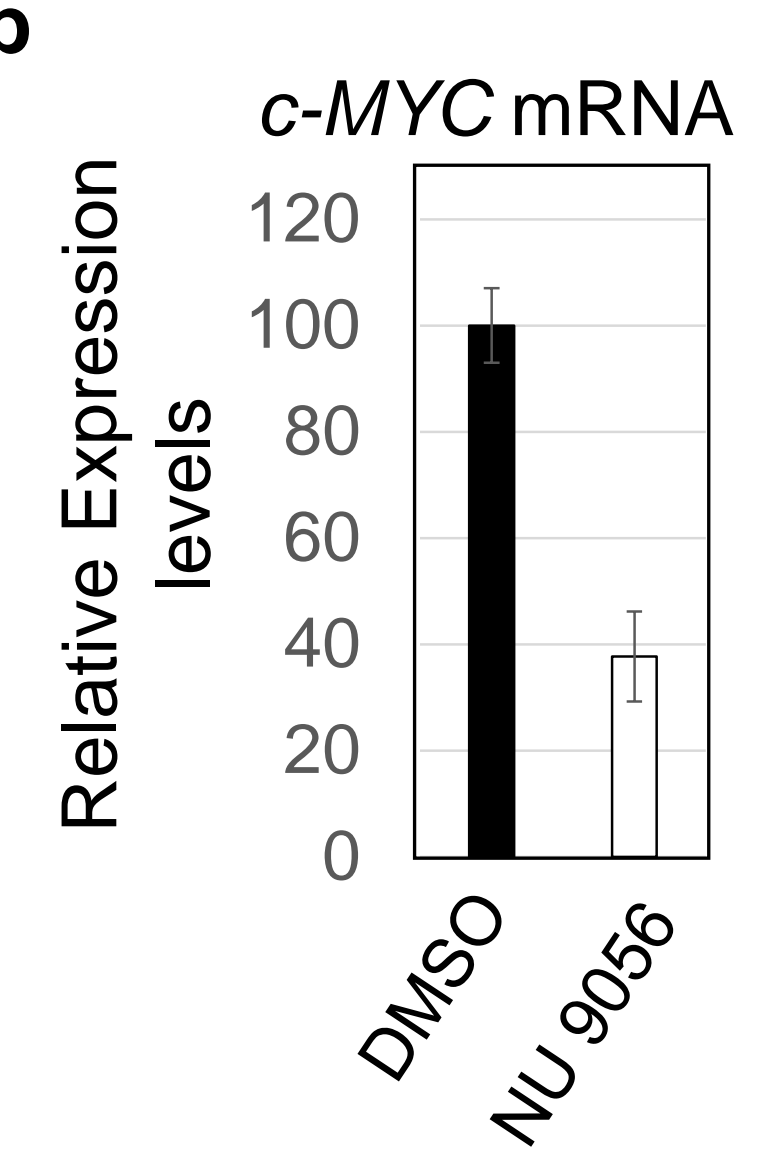

C
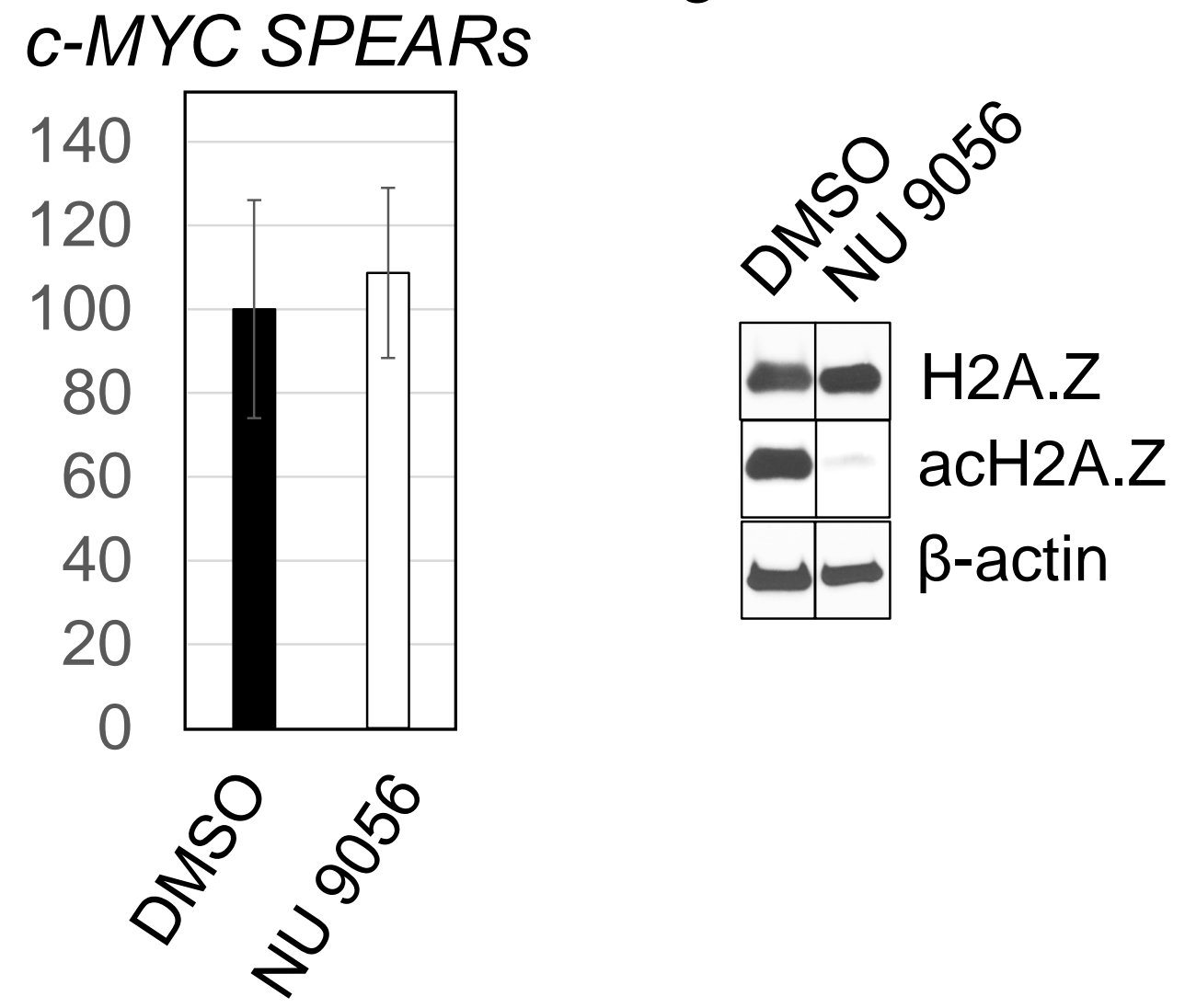

f Peptide AGGKAGKDSGKAKTK with Light Lysines and mixture of light and heavy acetyl groups on $\mathrm{K} 4, \mathrm{~K} 7$ and $\mathrm{K} 11$

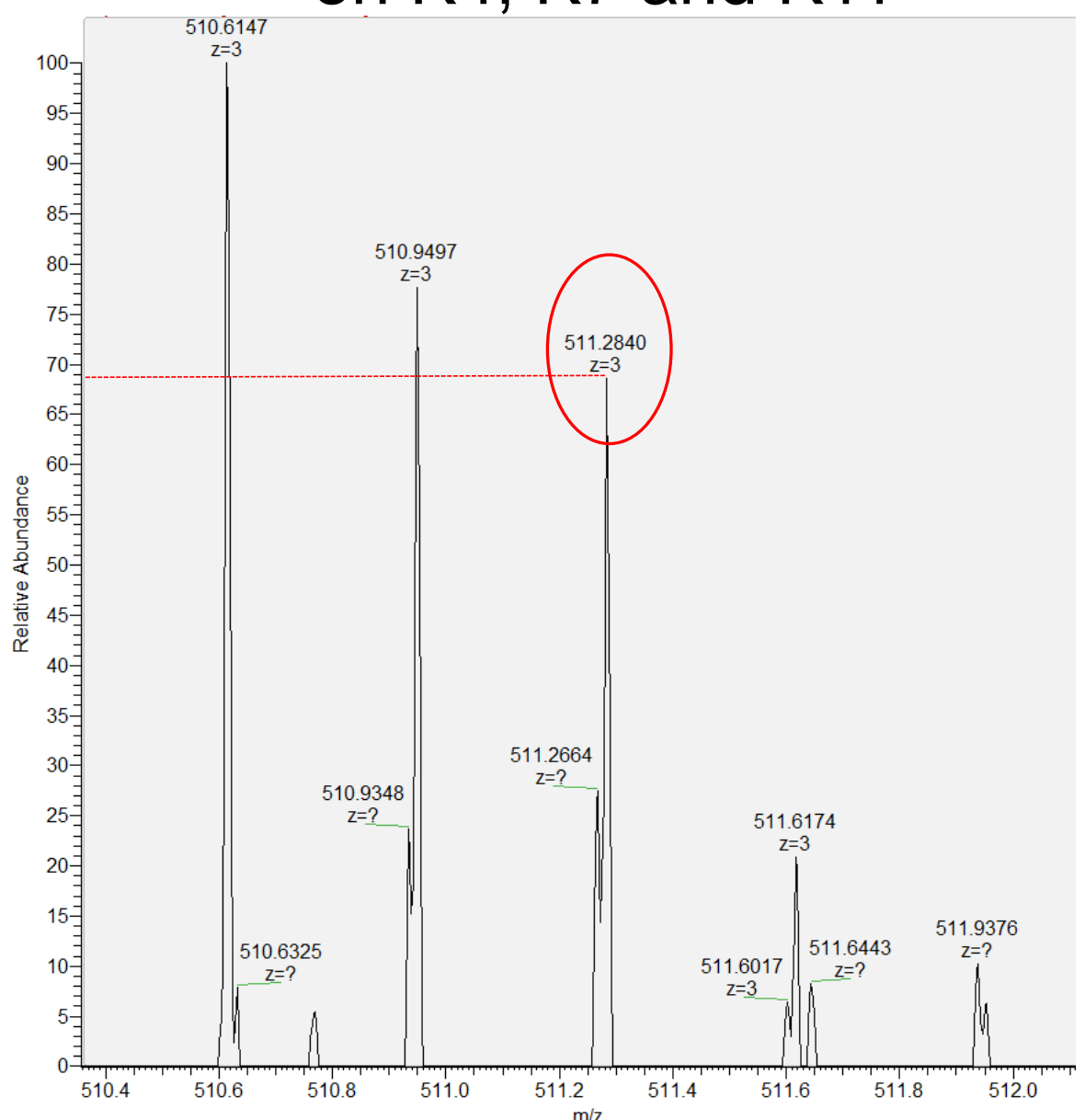

Sample "HATs Inhibitor NU 90564 hours" with Light Lysines and light acetyl groups on $\mathrm{K} 4, \mathrm{~K} 7$ and $\mathrm{K} 11$

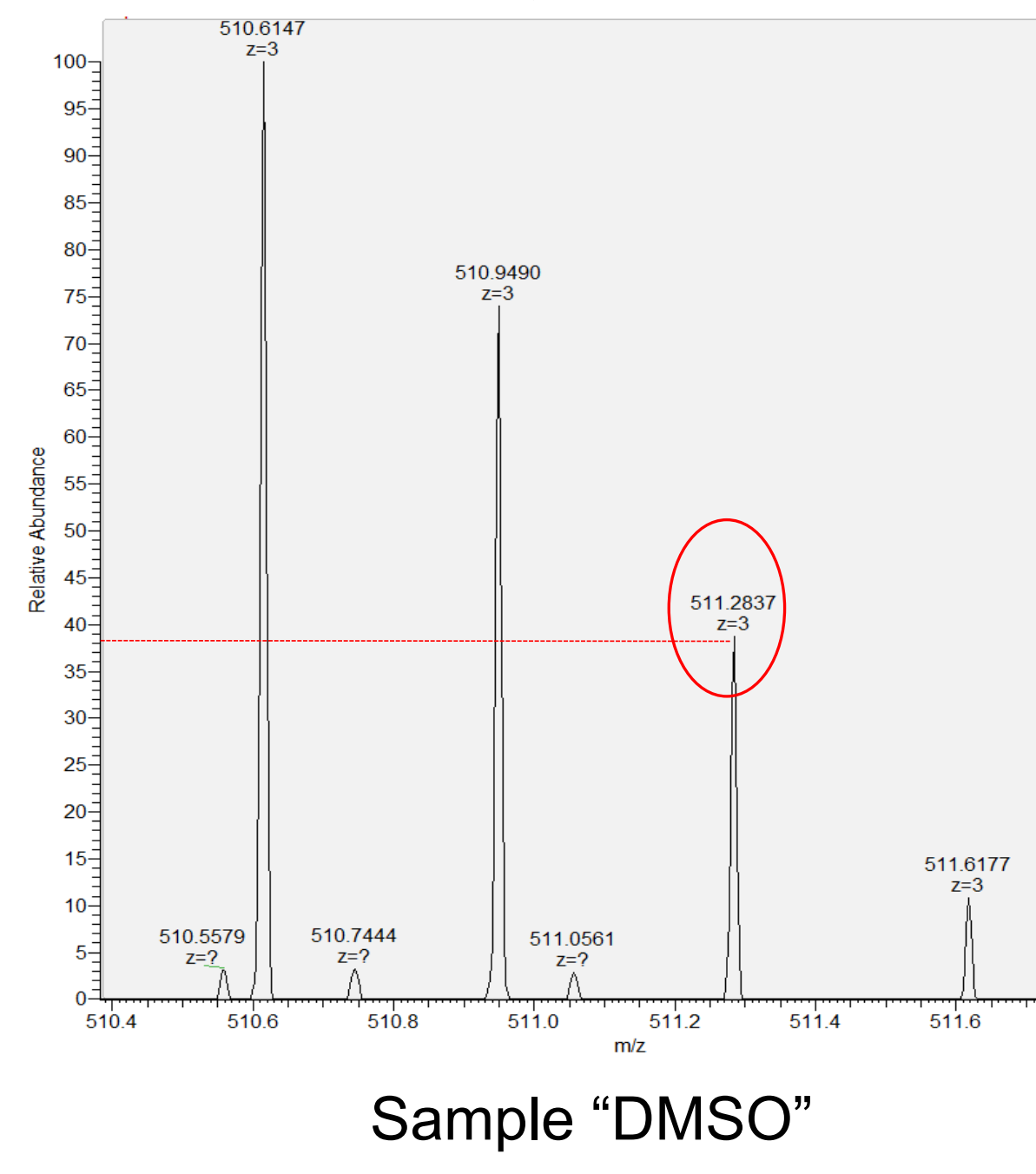

Ebralidze et al. Fig. 5 


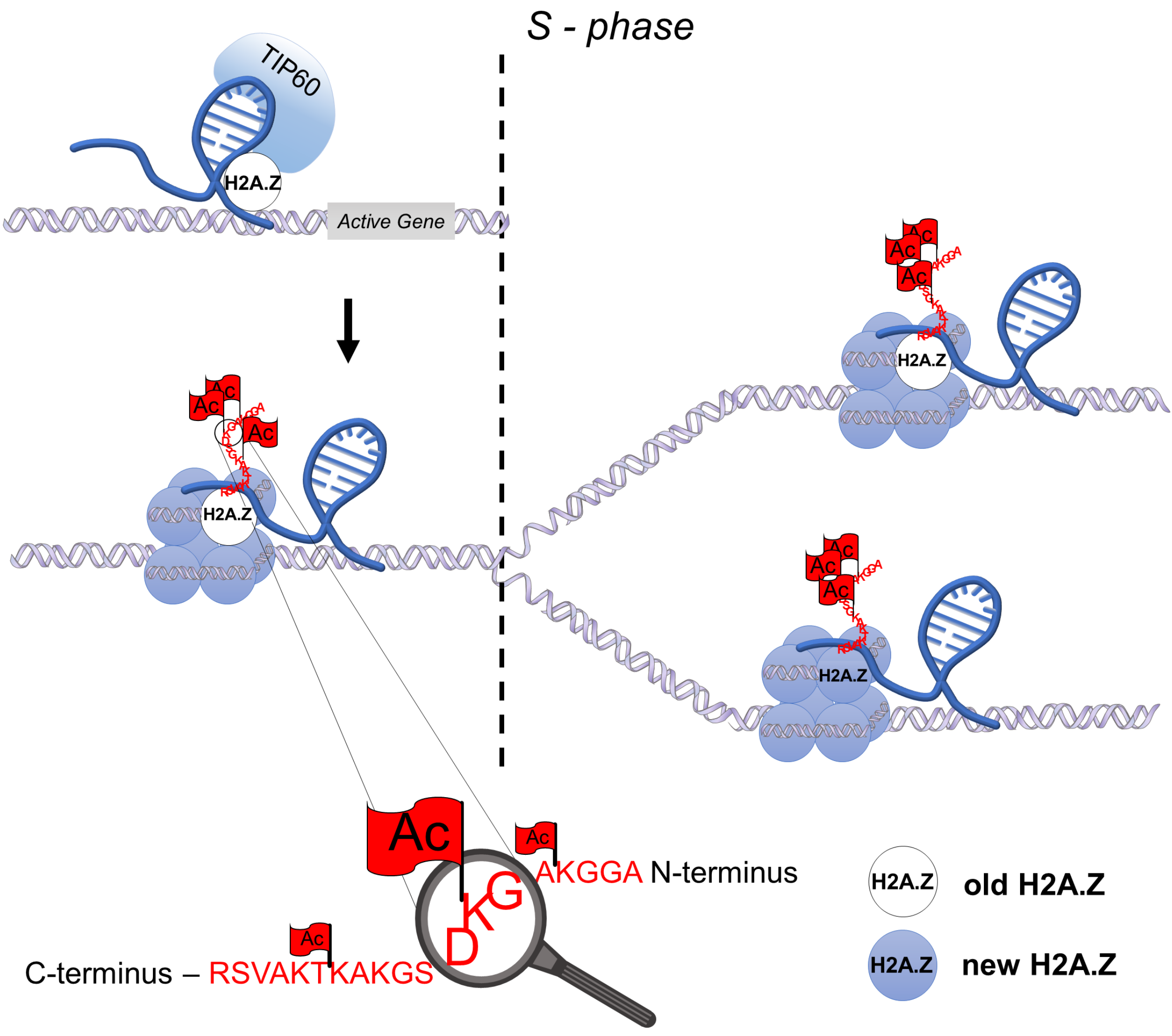

\section{Ebralidze et al. Fig. 6}

